\title{
Nonlinear Stochastic Integrals for Hyperfinite Lévy Processes
}

\author{
Tom Lindstrøm*
}

\begin{abstract}
We develop a notion of nonlinear stochastic integrals for hyperfinite Lévy processes, and use it to find exact formulas for expressions which are intuitively of the form $\sum_{s=0}^{t} \phi\left(\omega, d l_{s}, s\right)$ and $\prod_{s=0}^{t} \psi\left(\omega, d l_{s}, s\right)$, where $l$ is a Lévy process. These formulas are then applied to geometric Lévy processes, infinitesimal transformations of hyperfinite Lévy processes, and to minimal martingale measures.
\end{abstract}

Keywords: Stochastic integrals, Lévy processes, nonstandard analysis, hyperfinite Lévy processes, minimal martingale measures

AMS Subject Classification (2000): Primary 60G51, 60H05, Secondary 03H05, 28E05, 91B28

Consider a stochastic integral $\int X d M$ where $M$ is, say, an n-dimensional martingale and $X$ is a process taking values in the $m \times n$-matrices. The intuitive idea is that at each time $t$, the matrix $X(\omega, t)$ is multiplied by the increments $d M(\omega, t)$ of the martingale, and the results are then summed up to give the integral. Put a little more abstractly: at each time $t$ a linear function (represented by the matrix $X$ ) acts on the increments of $M$ and produces the increments of the integral process. In this paper I want to study what happens when the integrand $X$ acts on the increments in a more general (i.e. nonlinear) way.

Let me point out that "nonlinear" stochastic integrals in this sense have already been studied to some extent. In the stochastic analysis of Lévy processes as presented, e.g., in the recent books by Applebaum [3] and Øksendal and Sulem [12], the Lévy process is decomposed into a continuous part and a pure jump part. Stochastic integrals of the continuous part are treated in the traditional "linear" way, while the integrals of the pure jump part need not be linear. One of the motivations for this paper is that a fully nonlinear theory which puts the continuous part and the jump part on an equal footing, may be a more natural tool for applications in, e.g., mathematical finance, optimal stopping, and control theory - after all, we can not expect nature and society to

* Centre of Mathematics for Applications and Department of Mathematics, PO Box 1053 Blindern, N-0316 Oslo, Norway. e-mail:lindstro@math.uio.no 
deliver their processes in decomposed form! Another motivation is that nonlinear stochastic integrals turn out to be an excellent tool for producing interesting formulas. This is actually where the work on this paper started - I was simply looking for ways to understand and extend some of the fundamental formulas in [12], and the nonlinear stochastic integral turned out to be the unifying concept I needed in order to avoid doing (essentially) the same computations over and over again. Although I hope to turn to applications in the future, the emphasis of this paper is strictly on the second motivation, i.e., on mathematical formulas.

The main tool of the paper is nonstandard analysis and especially the theory of hyperfinite Lévy processes developed in [9]. In nonstandard theory, the increments $\Delta L_{t}$ of a process $L$ exist as concrete objects, and hence (nonlinear) operations on increments are trivial to define. The challenge is to show that they lead to finite processes which can be interpreted in a standard framework. I assume that the reader has a good general background in nonstandard probability theory, but begin the paper with brief reviews of the most relevant parts of the theory of hyperfinite Lévy processes (in Section 1) and the theory of stochastic integration with respect to nonstandard martingales (Section 2). Section 2 also includes some new results on (linear) stochastic integration with respect to hyperfinite Lévy processes.

The main part of the paper begins in Section 3 where nonlinear stochastic integrals are introduced and where we prove the main representation theorem 3.5. This theorem may be thought of as a Sum Formula for expressions of the form $\sum_{s=0}^{t} \phi(\omega, \Delta L(\omega, t), t)$, and in Section 4 we use exponentiation to transform it into a Product Formula for expressions of the form $\prod_{s=0}^{t} \psi(\omega, \Delta L(\omega, s), s)$ (see Theorem 4.1). To get a feeling for the main ideas of the paper, it may be wise to look quickly and informally through Sections 3 and 4 before reading the more technical parts of the first two sections.

In the last four sections, we show how the two basic formulas can be used in a variety of settings. In Section 5, we use the Product Formula to find an expression for geometric Lévy processes which generalizes the one in [12]. In Section 6 and 7 , we look at the how we can produce new hyperfinite Lévy processes from old by transforming increments and transition probabilities, respectively. In the first case, we use the Sum Formula to find an expression for the resulting process, and in the second case we use the Product Formula to find an expression for the density of the new measure with respect to the original. In the last section, we study minimal martingale measures for nonlinear stochastic integrals, and again we use the Product Formula to find an expression for the density.

Acknowledgement: It is a pleasure to thank Bernt Øksendal for the excellent lectures over his book with Agnès Sulem [12] that got this paper started.

\section{Review of hyperfinite Lévy processes}

In this section, I briefly review the theory of hyperfinite Lévy processes. Almost everything can be found in [9], but I have tried to arrange the material in a way 
that is optimal for the purposes of this paper. Other nonstandard approaches to Lévy processes can be found in the papers by Albeverio and Herzberg [2] and $\mathrm{Ng}[11]$.

Let $\Delta t$ be a positive infinitesimal, and choose $K \in^{*} \mathbf{N}$ so large that $K \Delta t$ is infinite. We shall use

$$
T=\{k \Delta t: k=0,1,2, \ldots, K\}
$$

as our timeline, and we shall work with internal processes $X: \Omega \times T \rightarrow{ }^{*} \mathbf{R}^{d}$. For convenience we shall always assume that the underlying probability space $(\Omega, \mathcal{F}, P)$ is hyperfinite, but this is not really essential. The Loeb measure of $P$ is denoted by $P_{L}$, and all a.e.-statements are with respect to $P_{L}$ unless otherwise stated.

If $X: \Omega \times T \rightarrow^{*} \mathbf{R}^{d}$ is an internal process, we shall write $\Delta X(\omega, s):=$ $X(\omega, s+\Delta t)-X(\omega, s)$ for the forward increment of $X$ at time $s \in T$. When we sum over elements of the timeline, we shall use the convention that

$$
\sum_{s=r}^{t} X(s)=X(r)+X(r+\Delta t)+X(r+2 \Delta t)+\ldots+X(t-\Delta t)
$$

hence $X(r)$ is included in the sum, but $X(t)$ is not. The same convention applies to products:

$$
\prod_{s=r}^{t} X(s)=X(r) \cdot X(r+\Delta t) \cdot X(r+2 \Delta t) \cdot \ldots \cdot X(t-\Delta t) .
$$

To describe a hyperfinite random walk, we specify a hyperfinite set $A$ of elements in ${ }^{*} \mathbf{R}^{d}$ and an internal set of positive numbers $\left\{p_{a}\right\}_{a \in A}$ in ${ }^{*} \mathbf{R}$ such that $\sum_{a \in A} p_{a}=1$. We call $A$ the set of increments and $\left\{p_{a}\right\}_{a \in A}$ the transition probabilities.

Definition 1.1 $A$ hyperfinite random walk with increments $A$ and transition probabilities $\left\{p_{a}\right\}_{a \in A}$ is an internal process $L: \Omega \times T \rightarrow{ }^{*} \mathbf{R}^{d}$ such that:

(i) $L(0)=0$.

(ii) The increments $\Delta L(0), \Delta L(\Delta t), \ldots, \Delta L(t), \ldots$ are ${ }^{*}$-independent.

(iii) All increments $\Delta L(t)$ have the distribution specified by $A$ and $\left\{p_{a}\right\}_{a \in A}$, i.e.

$$
P[\Delta L(\omega, t)=a]=p_{a}
$$

for all $t \in T$ and all $a \in A$.

Given a hyperfinite random walk $L$, we shall let $\left\{\mathcal{F}_{t}\right\}_{t \in T}$ be the internal filtration generated by $L$.

We define the drift vector $\mu_{L} \in{ }^{*} \mathbf{R}^{d}$ by

$$
\mu_{L}:=\frac{1}{\Delta t} E[\Delta L(0)]=\frac{1}{\Delta t} \sum_{a \in A} a p_{a}
$$


and note that

$$
E[L(t)]=E\left[\sum_{s=0}^{t} \Delta L(s)\right]=\frac{t}{\Delta t} E[\Delta L(0)]=\mu_{L} t
$$

This means that the process $M_{L}(t):=L(t)-\mu_{L} t$ is a martingale with respect to the filtration $\left\{\mathcal{F}_{t}\right\}_{t \in T}$ generated by $L$, and we thus have a natural decomposition

$$
L(t)=\mu_{L} t+M_{L}(t)
$$

in a drift term and a martingale term. We also introduce a nonnegative number $\sigma_{L} \in{ }^{*} \mathbf{R}^{d}$ by

$$
\sigma_{L}^{2}:=\frac{1}{\Delta t} E\left[|\Delta L(0)|^{2}\right]=\frac{1}{\Delta t} \sum_{a \in A}|a|^{2} p_{a}
$$

and note the following simple, but useful identity [9, Lemma 1.2] (the proof is just a simple calculation):

Lemma 1.2 For all $t \in T$

$$
E\left[|L(t)|^{2}\right]=\sigma_{L}^{2} t+\left|\mu_{L}\right|^{2} t(t-\Delta t)
$$

We shall be particularly interested in hyperfinite Lévy processes, i.e., hyperfinite random walks which are finite in the following sense:

Definition 1.3 Let $L$ be a hyperfinite random walk. We call $L$ a hyperfinite Lévy process if the set

$$
\{\omega \mid L(\omega, t) \text { is finite for all finite } t \in T\}
$$

has Loeb measure 1.

This definition is a little impractical as there is no obvious way to check that it is satisfied. However, the following, more useful characterization was proved in [9, Theorem 4.3]. We use the notation:

$$
q_{k}:=\frac{1}{\Delta t} \sum_{|a|>k} p_{a}
$$

Theorem 1.4 (Characterization of hyperfinite Lévy processes) $A$ hyperfinite random walk $L$ is a hyperfinite Lévy process if and only if the following three conditions are satisfied:

(i) $\frac{1}{\Delta t} \sum_{|a| \leq k} a p_{a}$ is finite for all finite and noninfinitesimal $k \in{ }^{*} \mathbf{R}$.

(ii) $\frac{1}{\Delta t} \sum_{|a| \leq k}|a|^{2} p_{a}$ is finite for all finite $k \in^{*} \mathbf{R}$.

(iii) $\lim _{k \rightarrow \infty}{ }^{\circ} q_{k}=0$ in the sense that for every $\epsilon \in \mathbf{R}_{+}$, there is an $N \in \mathbf{N}$ such that $q_{k}<\epsilon$ when $k \geq N$. 
Hyperfinite Lévy processes may have bad integrability properties, and it is often convenient to approximate them with processes which behave better under integration. A hyperfinite random walk has finite increments if all $a \in A$ are finite (note that since $A$ is internal, this means that there is an $N \in \mathbf{N}$ such that $|a| \leq N$ for all $a \in A$ ). For hyperfinite random walks with finite increments, the characterization above reduces to:

Corollary 1.5 [9, Corollary 2.4] A hyperfinite random walk L with finite increments is a hyperfinite Lévy process if and only if both $\mu_{L}=\frac{1}{\Delta t} \sum_{a \in A} a p_{a}$ and $\sigma_{L}^{2}=\frac{1}{\Delta t} \sum_{a \in A}|a|^{2} p_{a}$ are finite.

The next result gives us the integrability properties we need:

Theorem 1.6 [9, Theorem 2.3] Let L be a hyperfinite Lévy process with finite increments. Then $\left|L_{t}\right|^{p}$ is S-integrable for all finite $p \in{ }^{*} \mathbf{R}_{+}$and all finite $t \in T$.

If we combine this result with the decomposition $L(t)=\mu_{L} t+M_{L}(t)$ above, we get (see [9, Corollary 2.5]):

Corollary 1.7 A hyperfinite Lévy process $L$ with finite increments can be decomposed as

$$
L(t)=\mu_{L} t+M_{L}(t)
$$

where $\mu_{L} \in^{*} \mathbf{R}^{d}$ is finite and $M_{L}$ is a martingale such that $\left|M_{L}(t)\right|^{p}$ is $S$ integrable for all finite $t$ and all finite $p \in \in^{*} \mathbf{R}_{+}$. In particular, $M_{L}$ is an $S L^{2}$ martingale (in the terminology of [1], an $S L^{2}$-martingale is just an internal martingale such that $\left|M_{L}(t)\right|^{2}$ is S-integrable for all finite $\left.t\right)$.

As there is a well-developed theory for stochastic integration with respect to $\mathrm{SL}^{2}$-martingales, this corollary will in the next section be our key to stochastic integration with respect to hyperfinite Lévy processes. To extend integration from processes with finite increments to the general case, we need to know how general hyperfinite Lévy processes can be approximated by hyperfinite Lévy processes with finite increments. Introducing the truncated processes $L \leq k$ for $k \in \in^{*} \mathbf{R}_{+}$by

$$
L^{\leq k}(\omega, t)=\sum\{\Delta L(\omega, s): s<t \text { and }|\Delta L(\omega, s)| \leq k\}
$$

we have the following result which is a combination of Corollary 4.2 and (the proof of) Proposition 3.4 in [9].

Proposition 1.8 Assume that $L$ is a hyperfinite Lévy process. Then the truncated process $L^{\leq k}$ is a hyperfinite Lévy process for all noninfinitesimal $k>0$. Moreover, for each finite $t \in T$ and each $\epsilon \in \mathbf{R}_{+}$, there is a $k \in \mathbf{R}_{+}$such that

$$
P\left[\omega: L(\omega, s)=L^{\leq k}(\omega, s) \text { for all } s \leq t\right]>1-\epsilon
$$

Note that $L^{\leq k}$ need not be a hyperfinite Lévy process when $k$ is infinitesimal.

Here is a very simple, but useful consequence of the proposition above. 
Corollary 1.9 Assume that $L$ is a hyperfinite Lévy process and that $t \in T$ is finite. Then $\sum_{s=0}^{t}|\Delta L(s)|^{2}$ is finite $P_{L}$-a.e.

Proof: Assume first that $L$ has finite increments. Then

$$
E\left(\sum_{s=0}^{t}|\Delta L(s)|^{2}\right)=\frac{t}{\Delta t} \sum_{a \in A}|a|^{2} p_{a}=t \sigma_{L}^{2}
$$

is finite according to Corollary 1.5, and hence $\sum_{s=0}^{t}|\Delta L(s)|^{2}$ must be finite $P_{L}$-a.e. The result for general hyperfinite Lévy processes now follows from the proposition above.

We end this section with a few words on the notion of a splitting infinitesi$m a l$ for a hyperfinite Lévy process $L$. This notion played a central part in [9], and will play an equally important part here. The starting point is simply that for many purposes it is convenient to split our hyperfinite Lévy process $L$ in a continuous part and a jump part. The continuous part would ideally consist of all the infinitesimal increments of $L$, while the jump part would ideally consist of all the noninfinitesimal increments. Since it in general is impossible to split infinitesimals and noninfinitesimals in an internal way, we must compromise somewhat. The idea is that if we split the increments at a sufficiently large infinitesimal, then the infinitesimal contributions to the jump part will be insignificant. Here is the precise definition of what "sufficiently large" means in this context ([9, Definition 5.1]):

Definition 1.10 An infinitesimal $\eta$ is called a splitting infinitesimal for the hyperfinite Lévy process $L$ if

$$
S-\lim _{b \downarrow 0}\left(\frac{1}{\Delta t} \sum_{\eta \leq a \leq b}|a|^{2} p_{a}\right)=0
$$

where the limit means that for any standard $\epsilon \in \mathbf{R}_{+}$, there is a standard $\delta \in \mathbf{R}_{+}$ such that $\frac{1}{\Delta t} \sum_{\eta \leq|a| \leq b}|a|^{2} p_{a}<\epsilon$ whenever $0 \precsim b<\delta$.

It is easy to see that splitting infinitesimals always exist. Note also that (1) is equivalent to

$$
\frac{1}{\Delta t} \sum_{\eta \leq|a| \leq \epsilon}|a|^{2} p_{a} \approx 0 \quad \text { for all infinitesimal } \epsilon>\eta
$$

In [9, Theorem 5.3], splitting infinitesimals were used to prove a nonstandard version of the Lévy-Itô decomposition of a Lévy process into a continuous part and a pure jump part. In this paper, we shall use them in a similar (but simpler) way to decompose nonlinear stochastic integrals into a well-behaved "jump part" and an "integral part" which (although it does contain jumps) is easy to control. 


\section{Stochastic integration with respect to hyper- finite Lévy processes}

In this section, I shall briefly review the basic theory of nonstandard stochastic integration with respect to martingales and show how it can be adapted to hyperfinite Lévy processes. For the martingale theory, I shall mainly follow the presentations in the book [1] and the survey paper [8] — the original papers are [7] and [6]. Another nonstandard approach to stochastic integration with respect to Lévy processes can be found in [2].

From a purely nonstandard point of view, stochastic integrals are easy to define. If $X, M: \Omega \times T \rightarrow^{*} \mathbf{R}$ are two internal processes, we simply define the stochastic integral $\int X d M$ to be the process

$$
\int_{0}^{t} X d M:=\sum_{s=0}^{t} X(s) \Delta M(s)
$$

The problem is that in this generality, the stochastic integral is likely to be infinite even when the processes $X$ and $M$ are finite. To control the integral, it is usual to restrict to the case where $M$ is a (sufficiently integrable) martingale and $X$ is a (sufficiently integrable) nonanticipating process. Let us briefly review the basic theory.

An internal filtration $\left\{\mathcal{F}_{t}\right\}_{t \in T}$ on our hyperfinite probability space $\Omega$ is simply an increasing, internal sequence of algebras of subsets of $\Omega$. For each $t \in T$, $\mathcal{F}_{t}$ defines an equivalence relation $\sim_{t}$ on $\Omega$ by:

$$
\omega \sim_{t} \omega^{\prime} \Longleftrightarrow \forall A \in \mathcal{F}_{t}\left(\omega \in A \Longleftrightarrow \omega^{\prime} \in A\right)
$$

An internal process $X: \Omega \times T \rightarrow^{*} \mathbf{R}$ is nonanticipating with respect to the filtration $\mathcal{F}_{t}$ if for all $t \in T$

$$
\omega \sim_{t} \omega^{\prime} \Rightarrow X(\omega, t)=X\left(\omega^{\prime}, t\right)
$$

Since we are working with hyperfinite probability spaces, this is equivalent to saying that $X(\cdot . t)$ is $\mathcal{F}_{t}$-measurable, but the formulation above is often easier to use. Intuitively, nonanticipation means that $X$ can not see into the future; its values at time $t$ are based on what has happened up to that time.

Let $M: \Omega \times T \rightarrow^{*} \mathbf{R}$ be an internal process, and assume that $M$ is a martingale with respect to an internal filtration $\left(\Omega,\left\{\mathcal{F}_{t}\right\}, P\right)$ (this just means that $M$ is nonanticipating and that $E\left(\Delta M_{t} \mid \mathcal{F}_{t}\right)=0$ for all $\left.t\right)$. We call $M$ an $\mathrm{SL}^{2}$ martingale if $M_{t}^{2}$ is S-integrable for all finite $t \in T$. The $\mathrm{SL}^{2}$-martingales will be our basic class of integrator processes. To define the basic class of integrands, let $\nu_{M}$ be the internal measure on $\Omega \times T$ defined by

$$
\nu_{M}(A \times\{t\})=\int_{A} \Delta M(\omega, t)^{2} d P(\omega)
$$

for all measurable $A \subseteq \Omega$. We want our integrands to be S-square integrable with respect to this Doléans measure $\nu_{M}$. More precisely, we define: 
Definition 2.1 Assume that $M$ is an $S L^{2}$-martingale. The set $S L^{2}(M)$ consists of all internal processes $X: \Omega \times T \rightarrow^{*} \mathbf{R}$ such that:

(i) $X$ is nonanticipating (with respect to the filtration of $M$ )

(ii) $X$ is $S$-square integrable with respect to $\nu_{M}$

The following result shows that $\mathrm{SL}^{2}(M)$ is a natural class of integrands:

Theorem 2.2 [1, Proposition 4.4.4 and Theorem 4.2.15] Assume that $M$ is an $S L^{2}$-martingale and that $X \in S L^{2}(M)$. Then $\int X d M$ is an $S L^{2}$-martingale. If $M$ is $S$-continuous, so is $\int X d M$.

By using localizing sequences of stopping times, it is possible to extend stochastic integration to an even larger class of integrands $S L(M)$ (see [1]), but we shall not use this larger class here.

When we turn to hyperfinite Lévy processes, we shall have to integrate with respect to multidimensional martingales. If $M: \Omega \times T \rightarrow{ }^{*} \mathbf{R}^{d}$ is a martingale (i.e. each component is a martingale), and $X: \Omega \times T \rightarrow^{*} \mathbf{R}^{m \times d}$ is a matrixvalued, nonanticipating process, we define $\int X d M$ in the natural way:

$$
\int_{0}^{t} X d M=\sum_{s=0}^{t} X(s) \cdot \Delta M(s)
$$

where - denotes matrix multiplication. We say that $M$ is an $\mathrm{SL}^{2}$-martingale if each component is a (one-dimensional) $\mathrm{SL}^{2}$-martingale, and we say that $X \in S L^{2}(M)$ if each component of $X$ is in $S L^{2}$ of the component of $M$ is is integrated against (i.e. $X_{i, j} \in S L^{2}\left(M_{j}\right)$ ). Theorem 2.2 now extends to the multidimensional case in the obvious way.

Let us now return to our hyperfinite Lévy processes. If $L$ is a hyperfinite Lévy process with finite increments, we know from Corollary 1.7 that $L$ can be written

$$
L(t)=\mu_{L} t+M_{L}(t)
$$

where $\mu_{L} \in{ }^{*} \mathbf{R}^{d}$ is finite, and $M_{L}$ is an $\mathrm{SL}^{2}$-martingale. To control a stochastic integral $\int X d L$, we have to control $\sum X \Delta t$ in addition to $\int X d M_{L}$. Let $\lambda$ be the nonstandard version of the Lebesgue integral, i.e. $\lambda$ is defined on all internal subsets $A$ of the timeline $T$ by

$$
\lambda(A)=|A| \Delta t
$$

We say that an internal function $F: T \rightarrow{ }^{*} \mathbf{R}^{d}$ is S-integrable with respect to $\lambda$ on finite intervals if $F \cdot \chi_{[0, t]}$ is S-integrable with respect to $\lambda$ for all finite $t \in T$. We are now ready to define the set $I(L)$ of natural integrands with respect to a hyperfinite Lévy process $L$.

Definition 2.3 Let $L$ be a hyperfinite Lévy process with finite increments. The internal process $X$ belongs to the set $I(L)$ if the following two conditions are satisfied: 
(i) $X \in S L^{2}\left(M_{L}\right)$

(ii) the function $t \rightarrow X(\omega, t)$ is S-integrable with respect to $\lambda$ on finite intervals for $P_{L}$-almost all $\omega$.

If $L$ is a general hyperfinite Lévy process, we say that $X \in I(L)$ if $X \in I\left(L^{\leq k}\right)$ for all finite and noninfinitesimal $k \in{ }^{*} \mathbf{R}_{+}$.

The next result just shows that these definitions do what they are intended to do.

Proposition 2.4 If $L$ is a hyperfinite Lévy process and $X \in I(L)$, then there is a set $\Omega^{\prime} \subseteq \Omega$ of Loeb measure one such that $\int_{0}^{t} X d L$ is finite for all $\omega \in \Omega^{\prime}$ and all finite $t \in T$.

Proof: For all finite and noninfinitesimal $k \in^{*} \mathbf{R}_{+}$, decompose $L \leq k$ in a drift part and a martingale part: $L^{\leq k}(t)=\mu_{k} t+M_{k}(t)$. Observe that

$$
\int_{0}^{t} X d L^{\leq k}=\mu_{k} \int_{0}^{t} X d \lambda+\int_{0}^{t} X d M_{k}
$$

By the definition above, both terms on the right are finite for all $t$ on a set of Loeb measure one. The general result now follows from Proposition 1.8.

We end this section with two technical lemmas which will be needed in the next section.

Lemma 2.5 Assume that $L$ is a hyperfinite Lévy process, and that $G: \Omega \times T \rightarrow$ ${ }^{*} \mathbf{R}$ is nonanticipating. For any $\eta \in^{*} \mathbf{R}_{+}$, let $L_{i}^{\leq \eta}$ and $L_{j}^{\leq \eta}$ be two components of the truncated process $L^{\leq \eta}=\left(L_{1}^{\leq \eta}, L_{2}^{\leq \eta}, \ldots, L_{d}^{\leq \eta}\right)$. Then

$$
E\left(\sum_{s=0}^{t}\left|G(s) \Delta L_{i}^{\leq \eta}(s) \Delta L_{j}^{\leq \eta}(s)\right|\right) \leq \sigma_{\eta}^{2} E\left(\sum_{s=0}^{t}|G(s)| \Delta t\right) \leq \sigma_{L}^{2} E\left(\sum_{s=0}^{t}|G(s)| \Delta t\right)
$$

where $\sigma_{\eta}^{2}:=\sigma_{L \leq \eta}^{2}=\frac{1}{\Delta t} \sum_{a \leq \eta}|a|^{2} p_{a}$.

Proof: We have

$$
\begin{gathered}
E\left(\sum_{s=0}^{t}\left|G(s) \Delta L_{i}^{\leq \eta}(s) \Delta L_{j}^{\leq \eta}(s)\right|\right) \leq E\left(\sum_{s=0}^{t}\left|G(s) \| \Delta L^{\leq \eta}(s)\right|^{2}\right) \leq \\
\leq E\left(\sum_{s=0}^{t}|G(s)|\right) \sum_{|a| \leq \eta}|a|^{2} p_{a}=\sigma_{\eta}^{2} E\left(\sum_{s=0}^{t}|G(s)| \Delta t\right) \leq \sigma_{L}^{2} E\left(\sum_{s=0}^{t}|G(s)| \Delta t\right)
\end{gathered}
$$

where we have used that $G$ is nonanticipating to get from the second to the third term.

The second of our lemmas gives us more precise information about the sums $\sum G(s) \Delta L_{i}(s) \Delta L_{j}(s)$ in the case where $L$ has infinitesimal increments. We let $a_{i}$ denote the $i$-th component of the vector $a \in{ }^{*} \mathbf{R}^{d}$. 
Lemma 2.6 Assume that $L$ is a hyperfinite Lévy process with infinitesimal increments, and that $G: \Omega \times T \rightarrow^{*} \mathbf{R}$ is a nonanticipating process such that $G(\omega, t)|\Delta L(\omega, t)| \approx 0$ for all $\omega$ and all finite $t \in T$. Let

$$
C_{i, j}=\frac{1}{\Delta t} \sum_{a \in A} a_{i} a_{j} p_{a}=\frac{1}{\Delta t} E\left(\Delta L_{i}(t) \Delta L_{j}(t) \mid \mathcal{F}_{t}\right)
$$

Then there is a set $\Omega^{\prime} \subseteq \Omega$ of Loeb measure one such that

$$
\sum_{s=0}^{t} G(\omega, s) \Delta L_{i}(\omega, s) \Delta L_{j}(\omega, s) \approx \sum_{s=0}^{t} C_{i, j} G(\omega, s) \Delta t
$$

for all finite $t \in T$ and all $\omega \in \Omega^{\prime}$.

Proof: Define the process $N$ by

$$
N(t)=\sum_{s=0}^{t}\left(G(s) \Delta L_{i}(s) \Delta L_{j}(s)-C_{i, j} G(s) \Delta t\right)
$$

Observe that $N$ is a martingale since

$$
\begin{gathered}
E\left(\Delta N(s) \mid \mathcal{F}_{s}\right)=E\left(G(s) \Delta L_{i}(s) \Delta L_{j}(s)-C_{i, j} G(s) \Delta t \mid \mathcal{F}_{s}\right)= \\
=G(s) E\left(\Delta L_{i}(s) \Delta L_{j}(s) \mid \mathcal{F}_{s}\right)-C_{i, j} G(s) \Delta t= \\
=C_{i, j} G(s) \Delta t-C_{i, j} G(s) \Delta t=0
\end{gathered}
$$

We compute the expectation of the quadratic variation of $N$ :

$$
\begin{gathered}
E([N](t))=E\left(\sum_{s=0}^{t} \Delta N(s)^{2}\right)=E\left(\sum_{s=0}^{t}\left(G(s) \Delta L_{i}(s) \Delta L_{j}(s)-C_{i, j} G(s) \Delta t\right)^{2}\right)= \\
=E\left(\sum_{s=0}^{t}\left(G(s) \Delta L_{i}(s) \Delta L_{j}(s)\right)^{2}\right)-E\left(\sum_{s=0}^{t}\left(C_{i, j} G(s) \Delta t\right)^{2}\right) \leq \\
\leq E\left(\sum_{s=0}^{t}\left(G(s) \Delta L_{i}(s) \Delta L_{j}(s)\right)^{2}\right) \leq E\left(\sum_{s=0}^{t}(G(s)|\Delta L(s)|)^{2}|\Delta L(s)|^{2}\right) \approx 0
\end{gathered}
$$

where we in the last step have used the hypothesis $G(\omega, t)|\Delta L(\omega, t)| \approx 0$ plus the fact that $E\left(\sum_{s=0}^{t}|\Delta L(s)|^{2}\right)=t \sigma_{L}^{2}$ is finite to show that the expression is infinitesimal. Since a simple martingale identity tells us that $\left.E(N(t))^{2}\right)=$ $E([N](t))$, we have from Doob's inequality:

$$
E\left(\sup _{s \leq t} N(s)^{2}\right) \leq 4 E\left(N(t)^{2}\right)=4 E([N](t)) \approx 0
$$


and the lemma follows.

Remark In [9] the matrix $C^{L}=\left\{C_{i, j}\right\}$ is called the infinitesimal covariance matrix of $L$, and it is shown (Lemma 7.4) that $C^{L}$ is symmetric and nonnegative definite, and that

$$
\left\langle C^{L} x, x\right\rangle=\frac{1}{\Delta t} \sum_{a \in A}\langle a, x\rangle^{2} p_{a} \leq \sigma_{L}^{2}|x|^{2}
$$

where $\langle\cdot, \cdot\rangle$ is the inner product in $\mathbf{R}^{d}$.

\section{Nonlinear stochastic integrals}

We are now ready to turn to our main topic: nonlinear stochastic integrals. As indicated in the introduction, these are integrals where the integrand acts on the increments of the integrator process in a nonlinear way. Since the increments of a hyperfinite process are concrete and well-defined objects, nonlinear actions are trivial to define. The challenge is to prove that the resulting integrals are finite and well-behaved, or — put a little differently — to find conditions which guarantee that the integrals are finite and well-behaved. The main result of this section (the Sum Formula 3.5) shows that we have managed to find such conditions, and it also shows that the integral can be computed is a way that makes sense also from a standard perspective.

We start with a hyperfinite Lévy process $L$, let $\left\{\mathcal{F}_{t}\right\}_{t \in T}$ be the filtration generated by $L$, and let $\left\{\sim_{t}\right\}_{t \in T}$ be the equivalence relations generated by $\left\{\mathcal{F}_{t}\right\}_{t \in T}$. An internal function

$$
\phi: \Omega \times{ }^{*} \mathbf{R}^{d} \times T \rightarrow^{*} \mathbf{R}
$$

is called nonanticipating if

$$
\phi(\omega, x, t)=\phi(\tilde{\omega}, x, t)
$$

whenever $\omega \sim_{t} \tilde{\omega}$. The nonlinear stochastic integral (NSI) of $\phi$ with respect to $L$ is the process $I=\int \phi\left(\omega, d L_{s}, s\right)$ defined by

$$
I(\omega, t):=\sum_{s=0}^{t} \phi(\omega, \Delta L(s, \omega), s)
$$

We shall usually write

$$
I(\omega, t)=\int_{0}^{t} \phi\left(\omega, d L_{s}, s\right)
$$

Note that if $x \mapsto \phi(\omega, x, t)$ is linear (for all $t$ and $\omega$ ), then $I$ is a stochastic integral in the usual sense - hence the name nonlinear stochastic integral.

For the NSI $I$ to make standard sense, we have to impose conditions on the integrand $\phi$; e.g., it is clear that we need $\phi(\omega, \Delta L(s), s)$ to be infinitesimal for 
most times $s$. We shall assume that $\phi(\omega, 0, s)=0$ and in addition that $\phi$ is twice continuously differentiable in a (nonstandard) sense that we now describe.

Abusing notation slightly, we shall write $\frac{\partial}{\partial x_{i}}, \nabla, \frac{\partial^{2}}{\partial x_{i} \partial x_{j}}$ etc. for the nonstandard extensions of the differential operators $\frac{\partial}{\partial x_{i}}, \nabla, \frac{\partial^{2}}{\partial x_{i} \partial x_{j}}$. When we differentiate integrand functions $\phi(\omega, x, t)$, the derivatives are always with respct to the space variables $x=\left(x_{1}, x_{2}, \ldots, x_{d}\right)$ unless otherwise specified. We shall call an internal function $F:{ }^{*} \mathbf{R}^{d} \rightarrow{ }^{*} \mathbf{R}^{m}$ S-continuous if whenever $x, y$ are finite and infinitely close, then $F(x), F(y)$ are also finite and infinitely close (note the finiteness condition on $F(x), F(y)$ which is not always included in the definition of S-continuity).

Definition 3.1 For $r \in \mathbf{N}$, the space $S C^{r}\left({ }^{*} \mathbf{R}^{d},{ }^{*} \mathbf{R}^{m}\right)$ of $r$-times $S$-continuously differentiable functions from ${ }^{*} \mathbf{R}^{d}$ to ${ }^{*} \mathbf{R}^{m}$ consists of all internal functions $F$ : ${ }^{*} \mathbf{R}^{d} \rightarrow{ }^{*} \mathbf{R}^{m}$ such that $F$ and all its partial derivatives of order $r$ or less exist and are $S$-continuous.

With this preparation, we can introduce our space of integrands.

Definition 3.2 Let $L$ be a hyperfinite Lévy process. The set $N I(L)$ of nonlinear integrands of $L$ consists of all nonanticipating, internal maps $\phi: \Omega \times$ ${ }^{*} \mathbf{R}^{d} \times T \rightarrow{ }^{*} \mathbf{R}$ such that:

(i) $\phi(\omega, 0, t)=0$ for all $\omega$ and $t$.

(ii) There is a set $\Omega^{\prime} \subseteq \Omega$ of $P_{L}$-measure one such that $\phi(\omega, \cdot, t) \in S C^{2}\left({ }^{*} \mathbf{R}^{d},{ }^{*} \mathbf{R}\right)$ for all $\omega \in \Omega^{\prime}$ and all finite $t$.

(iii) $\nabla \phi(\omega, 0, t) \in I(L)$ (recall Definition 2.3).

In Section 1 we introduced the upper truncations $L^{\leq k}$ of our process $L$ by

$$
L^{\leq k}(\omega, t)=\sum\{\Delta L(\omega, s): s<t \text { and }|\Delta L(\omega, s)| \leq k\}
$$

We shall also need the lower truncations $L^{>k}$ defined by

$$
L^{>k}(\omega, t)=\sum\{\Delta L(\omega, s): s<t \text { and }|\Delta L(\omega, s)|>k\}
$$

Obviously,

$$
L_{t}=L_{t}^{>k}+L_{t}^{\leq k}
$$

We are now ready for the fundamental calculation of this paper. We assume that $\phi \in N I(L)$ and that $\eta$ is a splitting infinitesimal (recall Definition 1.10). The idea is to use $\eta$ to split the nonlinear integral in two parts - a jump part and an integral part - which can be controlled separately.

$$
\begin{gathered}
\int_{0}^{t} \phi(\omega, d L(s), s)=\sum_{s=0}^{t} \phi\left(\omega, \Delta L^{>\eta}(s), s\right)+\sum_{s=0}^{t} \phi\left(\omega, \Delta L^{\leq \eta}(s), s\right)= \\
=\sum_{s=0}^{t}\left\{\phi\left(\omega, \Delta L^{>\eta}(s), s\right)-\nabla \phi(\omega, 0, s) \cdot \Delta L^{>\eta}(s)\right\}+
\end{gathered}
$$




$$
+\sum_{s=0}^{t} \phi\left(\omega, \Delta L^{\leq \eta}(s), s\right)+\sum_{s=0}^{t} \nabla \phi(\omega, 0, s) \cdot \Delta L^{>\eta}(s)
$$

where we have subtracted and added the same term. This may look rather mysterious, but the point is that the subtracted term $\nabla \phi(\omega, 0, s) \cdot \Delta L^{>\eta}(s)$ will stabilize the original jump term $\phi\left(\omega, \Delta L^{>\eta}(s), s\right)$ in a way that will be made clear in Lemma 3.3 below. By Taylor's formula (remember that $\phi(\omega, 0, s)=0$ )

$$
\begin{gathered}
\phi\left(\omega, \Delta L^{\leq \eta}(s), s\right)=\nabla \phi(\omega, 0, s) \cdot \Delta L^{\leq \eta}(s)+ \\
+\frac{1}{2} \sum_{i, j} \frac{\partial^{2} \phi}{\partial x_{i} \partial x_{j}}(\omega, \Theta(s), s) \Delta L_{i}^{\leq \eta}(s) \Delta L_{j}^{\leq \eta}(s)
\end{gathered}
$$

where $\Theta(s)$ is on the line segment form 0 to $\Delta L^{\leq \eta}(s)$. If we substitute this into the expression above, we get

$$
\begin{aligned}
& \int_{0}^{t} \phi(\omega, d L(s), s)=\sum_{s=0}^{t}\left\{\phi\left(\omega, \Delta L^{>\eta}(s), s\right)-\nabla \phi(\omega, 0, s) \cdot \Delta L^{>\eta}(s)\right\}+ \\
& +\sum_{s=0}^{t} \nabla \phi(\omega, 0, s) \cdot \Delta L(s)+\frac{1}{2} \sum_{s=0}^{t} \frac{\partial^{2} \phi}{\partial x_{i} \partial x_{j}}(\omega, \Theta(s), s) \Delta L_{i}^{\leq \eta}(s) \Delta L_{j}^{\leq \eta}(s)
\end{aligned}
$$

In this expression, the second term on the right is finite since $\nabla(\omega, 0, s)$ is integrable with respect to $L$. The last term is close to the expression in Lemma 2.6 , and should be reasonably easy to control. We therefore turn our attention to the first expression which is the key to the whole argument:

Lemma 3.3 Assume that $L$ is a hyperfinite Lévy process and that $\phi \in N I(L)$. Fix a finite $t \in T$ and for each $r \in{ }^{*} \mathbf{R}_{+}$, define

$$
S_{r}(t)=\sum_{s=0}^{t}\left\{\phi\left(\omega, \Delta L^{>r}(s), s\right)-\nabla \phi(\omega, 0, s) \cdot \Delta L^{>r}(s)\right\}
$$

Then

(i) $S_{r}(t)$ is finite $P_{L}$-a.e. for all finite $r$.

(ii) If $\eta$ is a splitting infinitesimal, then for $P_{L}$-a.a. $\omega$

$$
S-\lim _{r \downarrow \eta} S_{r}(t)=S_{\eta}(t)
$$

where the limit means that for each $\epsilon \in \mathbf{R}_{+}$, there is a $\delta \in \mathbf{R}_{+}$such that $\left|S_{\eta}(t)-S_{r}(t)\right|<\epsilon$ whenever $\eta \leq r<\delta$. 
Proof: (i) By Proposition 1.8 it clearly suffices to prove this when $L$ has finite increments. By Taylor's formula

$$
\begin{aligned}
S_{r}(t) & =\sum_{\{s<t: r<|\Delta L(s)|\}}\{\phi(\omega, \Delta L(s), s)-\nabla \phi(\omega, 0, s) \cdot \Delta L(s)\}= \\
& =\frac{1}{2} \sum_{\{s<t: r<|\Delta L(s)|\}} \frac{\partial^{2} \phi}{\partial x_{i} \partial x_{j}}(\omega, \Theta(s), s) \Delta L_{i}(s) \Delta L_{j}(s)
\end{aligned}
$$

for some $\Theta(s)$ on the line segment from 0 to $\Delta L(s)$. Since $L$ has finite increments and $\phi \in N I(L)$,

$$
K_{\omega}:=\max _{0 \leq s \leq t}\left\{\frac{1}{2}\left|\frac{\partial^{2} \phi}{\partial x_{i} \partial x_{j}}(\omega, \Theta(s), s)\right|\right\}
$$

is finite for $P_{L}$-a.a. $\omega$. Hence

$$
\left|S_{r}(t)\right| \leq K_{\omega} \sum_{\{s<t: r<|\Delta L(s)|\}}|\Delta L(s)|^{2}
$$

is finite a.e. by corollary 1.9 .

(ii) Just as above we have

$$
\begin{gathered}
\left|S_{\eta}(t)-S_{r}(t)\right| \leq \sum_{\{s<t: \eta<|\Delta L(s)| \leq r\}}|\phi(\omega, \Delta L(s), s)-\nabla \phi(\omega, 0, s) \cdot \Delta L(s)|= \\
=\frac{1}{2} \sum_{\{s<t: \eta<|\Delta L(s)| \leq r\}}\left|\frac{\partial^{2} \phi}{\partial x_{i}}(\omega, \Theta(s), s) \Delta L_{i}(s) \Delta L_{j}(s)\right| \leq \\
\leq K_{\omega} \sum_{\{s<t: \eta<|\Delta L(s)| \leq r\}}|\Delta L(s)|^{2}
\end{gathered}
$$

Let $\Omega_{N}=\left\{\omega: K_{\omega} \leq N\right\}$ for $N \in \mathbf{N}$. Since $\left.\phi \in N I(L)\right), K_{\omega}$ is finite a.e., and hence $P_{L}\left(\Omega_{N}\right) \rightarrow 1$ as $n \rightarrow \infty$. Observe that

$$
\begin{gathered}
E\left(1_{\Omega_{N}} K_{\omega} \sum_{\{s<t: \eta<|\Delta L(s)| \leq r\}}|\Delta L(s)|^{2}\right) \leq \\
\leq N E\left(\sum_{\{s<t: \eta<|\Delta L(s)| \leq r\}}|\Delta L(s)|^{2}\right)=N \frac{t}{\Delta t} \sum_{\eta<|a| \leq r}|a|^{2} p_{a}
\end{gathered}
$$

By the definition of splitting infinitesimals, the standard part of the right hand side of this inequality goes to 0 as the standard part of $r$ goes to 0 , and hence the standard part of the left hand side decreases to zero almost everywhere. Letting $N$ go to infinity, we see that the standard part of the right hand side of 
(4) goes to zero $P_{L}$-a.e,, and hence $\mathrm{S}$ - $\lim _{r \downarrow \eta} S_{r}(t)=S_{\eta}(t) P_{L}$-a.e.

We need to do a little bookkeeping with the second order term in our expression for the nonlinear stochastic integral $\int \phi(\omega, d L(s), s)$.

Lemma 3.4 Assume that $L$ is a hyperfinite Lévy process, and that $\phi \in N I(L)$. Let $\eta$ be an infinitesimal, and let $C^{\eta}=\left\{C_{i, j}^{\eta}\right\}$ be the infinitesimal covariance matrix given by $C_{i, j}^{\eta}=\frac{1}{\Delta t} \sum_{|a| \leq \eta} a_{i} a_{j} p_{a}$ Assume further that for all $s$ and $\omega$, $\Theta(\omega, s)$ is on the line segment from 0 to $\Delta L(\omega, s)$. Then on a set of Loeb measure one

$$
\sum_{s=0}^{t} \frac{\partial^{2} \phi}{\partial x_{i} \partial x_{j}}(\omega, \Theta(s), s) \Delta L_{i}^{\leq \eta}(s) \Delta L_{j}^{\leq \eta}(s) \approx C_{i, j}^{\eta} \sum_{s=0}^{t} \frac{\partial^{2} \phi}{\partial x_{i} \partial x_{j}}(\omega, 0, s) \Delta t
$$

for all finite $t$, and the two expressions are finite.

Proof: By definition of $N I(L), \sum_{s=0}^{t} \frac{\partial^{2} \phi}{\partial x_{i} \partial x_{j}}(\omega, 0, s) \Delta t$ is finite a.e. By Lemma 2.6 (truncating $\frac{\partial^{2} \phi}{\partial x_{i} \partial x_{j}}$ at an infinite number if necessary) we know that

$$
\sum_{s=0}^{t} \frac{\partial^{2} \phi}{\partial x_{i} \partial x_{j}}(\omega, 0, s) \Delta L_{i}^{\leq \eta}(\omega, s) \Delta L_{j}^{\leq \eta}(\omega, s) \approx \sum_{s=0}^{t} C_{i, j} \frac{\partial^{2} \phi}{\partial x_{i} \partial x_{j}}(\omega, 0, s) \Delta t
$$

and hence all that remains to prove is that

$$
\begin{aligned}
& \sum_{s=0}^{t} \frac{\partial^{2} \phi}{\partial x_{i} \partial x_{j}}(\omega, \Theta(s), s) \Delta L_{i}^{\leq \eta}(s) \Delta L_{j}^{\leq \eta}(s) \approx \\
& \approx \sum_{s=0}^{t} \frac{\partial^{2} \phi}{\partial x_{i} \partial x_{j}}(\omega, 0, s) \Delta L_{i}^{\leq \eta}(\omega, s) \Delta L_{j}^{\leq \eta}(\omega, s)
\end{aligned}
$$

Since $\Theta(s)$ is infinitesimal whenever $\Delta L_{i}^{\leq \eta}(\omega, s) \Delta L_{j}^{\leq \eta}(\omega, s)$ is different from zero, the S-continuity of $\frac{\partial^{2} \phi}{\partial x_{i} \partial x_{j}}$ implies that for $P_{L}$-a.a. $\omega$, there is an infinitesimal $\epsilon(\omega)$ such that

$$
\begin{gathered}
\sum_{s=0}^{t}\left|\frac{\partial^{2} \phi}{\partial x_{i} \partial x_{j}}(\omega, \Theta(s), s)-\frac{\partial^{2} \phi}{\partial x_{i} \partial x_{j}}(\omega, 0, s)\right|\left|\Delta L_{i}^{\leq \eta}(\omega, s)\right|\left|\Delta L_{j}^{\leq \eta}(\omega, s)\right| \leq \\
\leq \sum_{s=0}^{t} \epsilon(\omega)\left|\Delta L_{i}^{\leq \eta}(\omega, s)\right|\left|\Delta L_{j}^{\leq \eta}(\omega, s)\right| \leq \epsilon(\omega) \sum_{s=0}^{t}|\Delta L(\omega, s)|^{2} \approx 0
\end{gathered}
$$

where we have used Corollary 1.9 in the last step. The lemma follows.

We may now sum up our results in a theorem (writing $\int_{0}^{t} \frac{\partial^{2} \phi}{\partial x_{i} \partial x_{j}}(\omega, 0, s) d s$ for the hyperfinite sum $\left.\sum_{s=0}^{t} \frac{\partial^{2} \phi}{\partial x_{i} \partial x_{j}}(\omega, 0, s) \Delta t\right)$ : 
Theorem 3.5 (Sum Formula) Assume that $L$ is a hyperfinite Lévy process, and that $\phi \in N I(L)$. Then for $P_{L^{-}}$a.a. $\omega$, the nonlinear stochastic integral $\int_{0}^{t} \phi(\omega, d L(s), s)$ is finite for all finite $t \in T$ and

$$
\begin{gathered}
\int_{0}^{t} \phi(\omega, d L(s), s) \approx \sum_{s=0}^{t}\left\{\phi\left(\omega, \Delta L^{>\eta}(s), s\right)-\nabla \phi(\omega, 0, s) \cdot \Delta L^{>\eta}(s)\right\}+ \\
+\int_{0}^{t} \nabla \phi(\omega, 0, s) \cdot d L(s)+\frac{1}{2} \sum_{1 \leq i . j \leq d} C_{i, j}^{\eta} \int_{0}^{t} \frac{\partial^{2} \phi}{\partial x_{i} \partial x_{j}}(\omega, 0, s) d s
\end{gathered}
$$

Here $\eta$ is any splitting infinitesimal, and $C^{\eta}=\left\{C_{i, j}^{\eta}\right\}$ is the infinitesimal covariance matrix $C_{i, j}^{\eta}=\frac{1}{\Delta t} \sum_{|a| \leq \eta} a_{i} a_{j} p_{a}$. Moreover,

$$
S_{r}(t)=\sum_{s=0}^{t}\left\{\phi\left(\omega, \Delta L^{>r}(s), s\right)-\nabla \phi(\omega, 0, s) \cdot \Delta L^{>r}(s)\right\}
$$

is finite for all finite $r$ and $t$, and if $\eta$ is a splitting infinitesimal, then $S_{\eta}(t)=$ $S-\lim _{r \downarrow \eta} S_{r}(t)$.

Proof: According to our basic calculation above

$$
\begin{aligned}
& \int_{0}^{t} \phi(\omega, d L(s), s)=\sum_{s=0}^{t}\left\{\phi\left(\omega, \Delta L^{>\eta}(s), s\right)-\nabla \phi(\omega, 0, s) \cdot \Delta L^{>\eta}(s)\right\}+ \\
& +\sum_{s=0}^{t} \nabla \phi(\omega, 0, s) \cdot \Delta L(s)+\frac{1}{2} \sum_{s=0}^{t} \frac{\partial^{2} \phi}{\partial x_{i} \partial x_{j}}(\omega, \Theta(s), s) \Delta L_{i}^{\leq \eta}(s) \Delta L_{j}^{\leq \eta}(s)
\end{aligned}
$$

In the expression on the right, the first term is finite a.e. by Lemma 3.3, the second is finite by the definition of $N I(L)$, and the third is finite and infinitely close to $\frac{1}{2} \sum_{1 \leq i . j \leq d} C_{i, j}^{\eta} \int_{0}^{t} \frac{\partial^{2} \phi}{\partial x_{i} \partial x_{j}}(\omega, 0, s) d s$ according to Lemma 3.4 . This proves the formula. The statements about $S_{r}(t)$ and $S_{\eta}(t)$ are just Lemma 3.3.

Remark: Note that (a nonstandard version of) Itô's formula follows immediately from the Sum Formula: To compute $F\left(L_{T}\right)$, just write

$$
F\left(L_{T}\right)=F(0)+\sum_{t=0}^{t}\left(F\left(L_{t}+\Delta L_{t}\right)-F\left(L_{t}\right)\right)
$$

and use the Sum Formula with $\phi(\omega, a, t)=F(L(\omega, t)+a)-F(L(\omega, t))$.

As an example, let us take a look at the simplest of all (truly) nonlinear stochastic integrals - the quadratic variation:

Example: For simplicity we assume that $L$ is one-dimensional. The quadratic variation $[L](t):=\sum_{s=0}^{t} \Delta L(s)^{2}$ is clearly a nonlinear stochastic integral corresponding to $\phi(x)=x^{2}$. Since $\phi^{\prime}(0)=0$ and $\phi^{\prime \prime}(0)=2$, the Sum Formula in this case reduces to 


$$
[L](t) \approx \sum_{s=0}^{t} \Delta L^{>\eta}(s)^{2}+C t
$$

for a (diffusion) constant $C$. Hence the quadratic variation equals (up to an infinitesimal) the sum of the square of all noninfinitesimal increments plus a diffusion term which is just a constant mulitiplum of time.

This paper is about nonstandard processes, and we shall not spend much time translating our results into standard language. At this point, however, it may be appropriate just to sketch the main connections. It was proved in [9] that any hyperfinite Lévy process induces a standard Lévy process $l$ as its (right) standard part, $l={ }^{\circ} L$. It was also proved that all standard Lévy processes $l$ can be obtained in this way (at least as long as we identify all Lévy processes with the same law). It is not difficult to prove that if $\phi$ satisfies natural conditions (we need, e.g., to require some regularity in $t$ ), then the right hand side of the sum formula above is infinitely close to the standard expression

$$
\begin{gathered}
\sum_{s=0}^{t}\left\{{ }^{\circ} \phi(\omega, \Delta l(s), s)-\nabla^{\circ} \phi(\omega, 0, s) \cdot \Delta l(s)\right\}+ \\
+\int_{0}^{t} \nabla^{\circ} \phi(\omega, 0, s) \cdot d l(s)+\frac{1}{2} \sum_{1 \leq i, j \leq d}{ }^{\circ} C_{i, j}^{\eta} \int_{0}^{t} \frac{\partial^{2}{ }^{\circ} \phi}{\partial x_{i} \partial x_{j}}(\omega, 0, s) d s
\end{gathered}
$$

where

$$
\begin{gathered}
\sum_{s=0}^{t}\left\{{ }^{\circ} \phi(\omega, \Delta l(s), s)-\nabla^{\circ} \phi(\omega, 0, s) \cdot \Delta l(s)\right\}:= \\
:=\lim _{\epsilon \downarrow 0} \sum_{\left\{s<t: \Delta l_{s}>\epsilon\right\}}\left\{{ }^{\circ} \phi(\omega, \Delta l(s), s)-\nabla^{\circ} \phi(\omega, 0, s) \cdot \Delta l(s)\right\}
\end{gathered}
$$

and where $\Delta l_{s}$ denotes the (standard) jumps of the process $l$. Note that all the terms in (5) makes standard sense, and hence this formula can be used as a starting point for a standard investigation of nonlinear stochastic integrals. In such an approach, it may be useful to think of a (standard) nonlinear stochastic integral $\int_{0}^{T} \phi\left(\omega, d l_{t}, t\right)$ as a sum $\sum\left(\phi\left(\omega, l_{t+\Delta t}, t+\Delta t\right)-\phi\left(\omega, l_{t}, t\right)\right)$, where $0, \Delta t, 2 \Delta t, \ldots, T$ is a partition of $[0, T]$ into small (standard) intervals, and then use Itô's formula on each little interval. The task is then to handle the convergence problems as $\Delta t \rightarrow 0$.

\section{The product formula}

The results in the previous section give us a way to calculate sums of the form $\sum_{s=0}^{t} \phi(\omega, \Delta L(s), s)$ where $\phi(\omega, 0, t)=0$. In this section we shall take a look at 
the corresponding products $\prod_{s=0}^{t} \psi(\omega, \Delta L(s), s)$ where $\psi(\omega, 0, t)=1$. It is, of course, easy to turn products into sums by exponentiating:

$$
\prod_{s=0}^{t} \psi(\omega, \Delta L(s), s)=\prod_{s=0}^{t} \operatorname{sgn}(\psi(\omega, \Delta L(s), s)) e^{\sum_{s=0}^{t} \ln |\psi(\omega, \Delta L(s), s)|}
$$

(for the time being we just ignore the problems that occur when $\psi(\omega, \Delta L(s), s) \approx$ $0)$. If we let

$$
N(\omega, t):=|\{s<t: \psi(\omega, \Delta L(s), s)<0\}|
$$

be the number of times $\psi(\omega, \Delta L(s), s)$ is negative before time $t$, and assume that

$$
\phi(\omega, x, s):=\ln |\psi(\omega, x . s)|
$$

is in $N I(L)$, then by the Sum Formula 3.5:

$$
\begin{gathered}
\prod_{s=0}^{t} \psi(\omega, \Delta L(s), s) \approx \\
\approx(-1)^{N(\omega, t)} \exp \left(\sum_{s=0}^{t}\left\{\phi\left(\omega, \Delta L^{>\eta}(s), s\right)-\nabla \phi(\omega, 0, s) \cdot \Delta L^{>\eta}(s)\right\}+\right. \\
\left.+\int_{0}^{t} \nabla \phi(\omega, 0, s) \cdot d L(s)+\frac{1}{2} \sum_{1 \leq i, j \leq d} C_{i, j}^{\eta} \int_{0}^{t} \frac{\partial^{2} \phi}{\partial x_{i} \partial x_{j}}(\omega, 0, s) d s\right)= \\
=\left(\prod_{s=0}^{t} \psi\left(\omega, \Delta L^{>\eta}(s), s\right) e^{-\nabla \phi(\omega, 0, s) \cdot \Delta L^{>\eta}(s)}\right) \times \\
\times \exp \left(\int_{0}^{t} \nabla \phi(\omega, 0, s) \cdot d L(s)+\frac{1}{2} \sum_{1 \leq i, j \leq d} C_{i, j}^{\eta} \int_{0}^{t} \frac{\partial^{2} \phi}{\partial x_{i} \partial x_{j}}(\omega, 0, s) d s\right)
\end{gathered}
$$

where

$$
\begin{gathered}
\prod_{s=0}^{t} \psi\left(\omega, \Delta L^{>\eta}(s), s\right) e^{-\nabla \phi(\omega, 0, s) \cdot \Delta L^{>\eta}(s)}= \\
=S-\lim _{r \downarrow 0} \prod_{s=0}^{t} \psi\left(\omega, \Delta L^{>r}(s), s\right) e^{-\nabla \phi(\omega, 0, s) \cdot \Delta L^{>r}(s)}
\end{gathered}
$$

for $r \in \mathbf{R}_{+}$(the exponential term is needed for convergence). To express this relationship in terms of the original function $\psi$, we use that since $\phi(\omega, x, t)=$ $\ln |\psi(\omega, x, t)|$, we have

$$
\frac{\partial \phi}{\partial x_{i}}=\frac{\frac{\partial \psi}{\partial x_{i}}}{\psi}
$$


and

$$
\frac{\partial^{2} \phi}{\partial x_{i} \partial x_{j}}=\frac{\frac{\partial^{2} \psi}{\partial x_{i} \partial x_{j}} \psi-\frac{\partial \psi}{\partial x_{i}} \frac{\partial \psi}{\partial x_{j}}}{\psi^{2}}
$$

Since $\psi(\omega, 0, t)=1$, we get

$$
\frac{\partial \phi}{\partial x_{i}}(\omega, 0, t)=\frac{\partial \psi}{\partial x_{i}}(\omega, 0, t)
$$

and

$$
\frac{\partial^{2} \phi}{\partial x_{i} \partial x_{j}}(\omega, 0, t)=\left[\frac{\partial^{2} \psi}{\partial x_{i} \partial x_{j}}-\frac{\partial \psi}{\partial x_{i}} \frac{\partial \psi}{\partial x_{j}}\right](\omega, 0, t)
$$

If we substitute this into the formula above, we get

$$
\begin{gathered}
\prod_{s=0}^{t} \psi(\omega, \Delta L(s), s) \approx \\
\approx\left(\prod_{s=0}^{t} \psi\left(\omega, \Delta L^{>\eta}(s), s\right) e^{-\nabla \psi(\omega, 0, s) \cdot \Delta L^{>\eta}(s)}\right) \times \\
\times \exp \left(\int_{0}^{t} \nabla \psi(\omega, 0, s) \cdot d L(s)+\frac{1}{2} \sum_{i, j=1}^{d} C_{i, j}^{\eta} \int_{0}^{t}\left[\frac{\partial^{2} \psi}{\partial x_{i} \partial x_{j}}-\frac{\partial \psi}{\partial x_{i}} \frac{\partial \psi}{\partial x_{j}}\right](\omega, 0, s) d s\right)
\end{gathered}
$$

So far our calculations are quite formal, and we have neglected the problems which occur when $\psi\left(\omega, \Delta L_{s}, s\right)$ is close to zero. The next theorem takes care of the necessary bookkeeping.

Theorem 4.1 (Product Formula) Assume that $L$ is a hyperfinite Lévy process, and that the internal function $\psi: \Omega \times{ }^{*} \mathbf{R}^{d} \times T \rightarrow{ }^{*} \mathbf{R}$ satisfies the following conditions:

(i) $\psi(\omega, 0, t)=1$ for all $\omega$ and $t$.

(ii) There is a set $\Omega^{\prime} \subseteq \Omega$ of $P_{L}$-measure one such that $\psi(\omega, \cdot, t) \in S C^{2}\left({ }^{*} \mathbf{R}^{d},{ }^{*} \mathbf{R}\right)$ for all $\omega \in \Omega^{\prime}$ and all finite $t$.

(iii) $\nabla \psi(\omega, 0, t) \in I(L)$

Then for $P_{L}$-a.a. $\omega$, the product $\prod_{0}^{t} \psi(\omega, d L(s), s)$ is finite for all finite $t \in T$ and

$$
\begin{gathered}
\prod_{s=0}^{t} \psi(\omega, \Delta L(s), s) \approx \\
\approx\left(\prod_{s=0}^{t} \psi\left(\omega, \Delta L^{>\eta}(s), s\right) e^{-\nabla \psi(\omega, 0, s) \cdot \Delta L^{>\eta}(s)}\right) \times \\
\times \exp \left(\int_{0}^{t} \nabla \psi(\omega, 0, s) \cdot d L(s)+\frac{1}{2} \sum_{i, j=1}^{d} C_{i, j}^{\eta} \int_{0}^{t}\left[\frac{\partial^{2} \psi}{\partial x_{i} \partial x_{j}}-\frac{\partial \psi}{\partial x_{i}} \frac{\partial \psi}{\partial x_{j}}\right](\omega, 0, s) d s\right)
\end{gathered}
$$


Here $\eta$ is any splitting infinitesimal, and $C^{\eta}=\left\{C_{i, j}^{\eta}\right\}$ is the infinitesimal covariance matrix $C_{i, j}^{\eta}=\frac{1}{\Delta t} \sum_{|a| \leq \eta} a_{i} a_{j} p_{a}$. Moreover, the product on the right hand side is finite and given by

$$
\begin{gathered}
\prod_{s=0}^{t} \psi\left(\omega, \Delta L^{>\eta}(s), s\right) e^{-\nabla \phi(\omega, 0, s) \cdot \Delta L^{>\eta}(s)}= \\
=S-\lim _{r \downarrow \eta}\left(\prod_{s=0}^{t} \psi\left(\omega, \Delta L^{>r}(s), s\right) e^{-\nabla \phi(\omega, 0, s) \cdot \Delta L^{>r}(s)}\right)
\end{gathered}
$$

for $r \in \mathbf{R}_{+}$.

Proof: For each natural number $n>1$, we let $\log _{n}: \mathbf{R} \rightarrow \mathbf{R}$ be a lower bounded approximation to log. More precisely, we let $\log _{n}$ be an even function, bounded from below, with bounded and continuous first and second derivatives, and assume that $\log _{n}$ agrees with $\log (|\cdot|)$ on the set $\left\{x:|x| \geq \frac{1}{n}\right\}$. Assume also $\log _{n} x \geq \log |x|$ for all $x$ and that the sequence $\left\{\log _{n}\right\}$ is decreasing. Abusing notation slightly, we shall write $\log _{N}$, where $N \in^{*} \mathbf{N}$, for the elements in the nonstandard extension of the sequence $\left\{\log _{n}\right\}_{n \in \mathbf{N}}$.

Let $\Pi(\omega, t)=\prod_{s=0}^{t} \psi\left(\omega, \Delta L_{s}, s\right)$ and define

$$
\Pi_{n}(\omega, t)=(-1)^{N(\omega, t)} e^{\sum_{s=0}^{t} \phi_{n}\left(\omega, \Delta L_{s}, s\right)}
$$

(recall that $N(\omega, t)$ counts the number of times $\psi\left(\omega, \Delta L_{s}, s\right)$ is negative before time $t)$. Note that since $\log _{n} x \geq \log |x|$, we have $|\Pi(\omega, t)| \leq\left|\Pi_{n}(\omega, t)\right|$. Since the function $\phi_{n}=\log _{n} \circ \psi$ is in $N I(L)$ for all $n \in \mathbf{N}$, the Sum Formula 3.5 tells us that $\Pi_{n}(\omega, t)$ is finite almost everywhere and hence $\Pi(\omega, t)$ is finite almost everywhere. The Sum Formula also tells us that

$$
\begin{gathered}
\int_{0}^{t} \phi_{n}(\omega, d L(s), s) \approx \sum_{s=0}^{t}\left\{\phi_{n}\left(\omega, \Delta L^{>\eta}(s), s\right)-\nabla \phi_{n}(\omega, 0, s) \cdot \Delta L^{>\eta}(s)\right\}+ \\
+\int_{0}^{t} \nabla \phi_{n}(\omega, 0, s) \cdot d L(s)+\frac{1}{2} \sum_{i, j=1}^{d} C_{i, j}^{\eta} \int_{0}^{t} \frac{\partial^{2} \phi_{n}}{\partial x_{i} \partial x_{j}}(\omega, 0, s) d s= \\
\quad=\sum_{s=0}^{t}\left\{\phi_{n}\left(\omega, \Delta L^{>\eta}(s), s\right)-\nabla \psi(\omega, 0, s) \cdot \Delta L^{>\eta}(s)\right\}+ \\
+\int_{0}^{t} \nabla \psi(\omega, 0, s) \cdot d L(s)+\frac{1}{2} \sum_{i, j=1}^{d} C_{i, j}^{\eta} \int_{0}^{t}\left[\frac{\partial^{2} \psi}{\partial x_{i} \partial x_{j}}-\frac{\partial \psi}{\partial x_{i}} \frac{\partial \psi}{\partial x_{j}}\right](\omega, 0, s) d s
\end{gathered}
$$

where we in the last step have done the same calculations as above (recall that $\log _{n}$ locally looks like $\left.\log (|\cdot|)\right)$. Exponentiating, we get 


$$
\begin{gathered}
\Pi_{n}(\omega, t)=(-1)^{N(\omega, t)} \prod_{s=0}^{t} e^{\phi_{n}(\omega, d L(s), s)} \approx \\
\approx(-1)^{N(\omega, t)}\left(\prod_{s=0}^{t} e^{\phi_{n}\left(\omega, \Delta L^{>\eta}(s), s\right)-\nabla \psi(\omega, 0, s) \cdot \Delta L^{>\eta}(s)}\right) \times \\
\times \exp \left(\int_{0}^{t} \nabla \psi(\omega, 0, s) \cdot d L(s)+\frac{1}{2} \sum_{i, j=1}^{d} C_{i, j}^{\eta} \int_{0}^{t}\left[\frac{\partial^{2} \psi}{\partial x_{i} \partial x_{j}}-\frac{\partial \psi}{\partial x_{i}} \frac{\partial \psi}{\partial x_{j}}\right](\omega, 0, s) d s\right)
\end{gathered}
$$

Hence for all $n \in \mathbf{N}$, the following statement holds

$$
P\left[\omega \in \Omega \mid \forall t \leq n\left(\left|\Pi_{n}(\omega, t)-R_{n}(\omega, t)\right|<1 / n\right)\right]>1-\frac{1}{n}
$$

where $R_{n}(\omega, t)$ is the right hand side of the formula above. By overflow, the statement must also hold for some infinite $N \in^{*} \mathbf{N} \backslash \mathbf{N}$, and hence

$$
\begin{gathered}
\Pi_{N}(\omega, t)=(-1)^{N(\omega, t)} \prod_{s=0}^{t} e^{\phi_{N}(\omega, d L(s), s)} \approx \\
\approx(-1)^{N(\omega, t)}\left(\prod_{s=0}^{t} e^{\phi_{N}\left(\omega, \Delta L^{>\eta}(s), s\right)-\nabla \psi(\omega, 0, s) \cdot \Delta L^{>\eta}(s)}\right) \times \\
\times \exp \left(\int_{0}^{t} \nabla \psi(\omega, 0, s) \cdot d L(s)+\frac{1}{2} \sum_{i, j=1}^{d} C_{i, j}^{\eta} \int_{0}^{t}\left[\frac{\partial^{2} \psi}{\partial x_{i} \partial x_{j}}-\frac{\partial \psi}{\partial x_{i}} \frac{\partial \psi}{\partial x_{j}}\right](\omega, 0, s) d s\right)
\end{gathered}
$$

on a set of $P_{L}$-measure one.

Comparing the left and the right hand side of this formula to the left and the right hand side of the first formula in the theorem, respectively, we see that the terms agree except possibly when there is an $s<t$ such that $|\psi(\omega, \Delta L(s), s)|<$ $\frac{1}{N}$. But in that case both sides of the formula we want to prove are infinitesimal a.s., and hence the formula still holds. (To see this, note that if the product $\prod_{s=0}^{t} \psi\left(\omega, \Delta L_{s}, s\right)$ contains an infinitesimal factor, but is not itself infinitesimal, then for a finite choice of $n, \Pi_{n}(\omega, t)$ has to be infinite, and we know that this happens with probability zero).

It remains to prove the second formula in the theorem. Note first that since

$$
\begin{aligned}
& \prod_{s=0}^{t}\left|\psi\left(\omega, \Delta L^{>\eta}(s), s\right) e^{-\nabla \phi(\omega, 0, s) \cdot \Delta L^{>\eta}(s)}\right| \leq \\
& \quad \leq \prod_{s=0}^{t} e^{\phi_{n}\left(\omega, \Delta L^{>\eta}(s), s\right)-\nabla \psi(\omega, 0, s) \cdot \Delta L^{>\eta}(s)}
\end{aligned}
$$


for $n \in \mathbf{N}$, the first product must be finite since the second one is. Observe also that for finite $n>1$

$$
\frac{\prod_{s=0}^{t} \psi\left(\omega, \Delta L^{>\eta}(s), s\right) e^{-\nabla \phi(\omega, 0, s) \cdot \Delta L^{>\eta}(s)}}{\prod_{s=0}^{t} \psi\left(\omega, \Delta L^{>r}(s), s\right) e^{-\nabla \phi(\omega, 0, s) \cdot \Delta L^{>r}(s)}}
$$

equals

$$
\frac{\prod_{s=0}^{t} e^{\phi_{n}\left(\omega, \Delta L^{>\eta}(s), s\right)-\nabla \psi(\omega, 0, s) \cdot \Delta L^{>\eta}(s)}}{\prod_{s=0}^{t} e^{\phi_{n}\left(\omega, \Delta L^{>r}(s), s\right)-\nabla \psi(\omega, 0, s) \cdot \Delta L^{>r}(s)}}
$$

for all infinitesimal $r>\eta$ (the terms that do not cancel belong to jumps in the interval $(\eta, r]$, and $\log$ and $\log _{n}$ can not distinguish between these). The Sum Theorem tells us that the second fraction is infinitely close to one, and hence

$$
\begin{aligned}
& \prod_{s=0}^{t} \psi\left(\omega, \Delta L^{>\eta}(s), s\right) e^{-\nabla \phi(\omega, 0, s) \cdot \Delta L^{>\eta}(s)} \approx \\
& \approx \prod_{s=0}^{t} \psi\left(\omega, \Delta L^{>r}(s), s\right) e^{-\nabla \phi(\omega, 0, s) \cdot \Delta L^{>r}(s)}
\end{aligned}
$$

This is just a nonstandard version of the limit statement in the theorem.

Note that we may "standardize" the Product Formula the same way we "standardized" the Sum Formula at the end of Section 3. What we then get looks like a generalization of the expression for the stochastic exponential (see, e.g., [13, Theorem 37]). In fact, we get (a nonstandard) version of the stochastic exponential by applying the (one-dimensional) Product Formula to the function $\psi(\omega, x, s)=(1+x)$ (see the next section for more information on a closely related topic).

In the remainder of this paper, we shall look at various applications of nonlinear stochastic integrals and the Product Formula. We begin with an application of the Product Formula.

\section{$5 \quad$ Geometric Lévy processes}

In [12] a geometric Lévy process is defined as the solution of a stochastic differential equation of the form

$$
d x_{t}=x_{t}\left(\alpha d t+\beta d b_{t}+\gamma\left(\omega, d l_{t}, t\right)\right)
$$

where $l$ is a pure jump Lévy process, $b$ is a (standard) Brownian motion independent of $l$, the coefficients $\alpha, \beta$ are constants, and $\gamma$ is an adapted process satisfying the appropriate growth conditions. Since $l$ is a pure jump process, the (nonlinear) integral $\int \gamma\left(\omega, d l_{t}, t\right)$ can be defined, e.g., as a sum of jumps. For notational convenience we shall assume that $l$ is one-dimensional although the arguments work equally well in higher dimensions. 
Using Itô calculus it is shown in [12] that provided $\gamma(t, z) \geq-1$, we have

$$
\begin{aligned}
x(t) & =x(0) \exp \left\{\left(\alpha-\frac{1}{2} \beta^{2}\right) t+\beta b(t)+\right. \\
& +\int_{0}^{t} \int_{|z|<R}\{\ln (1+\gamma(s, z))-\gamma(s, z)\} \nu(d z) d s \\
& \left.+\int_{0}^{t} \int_{\mathbf{R}} \ln (1+\gamma(s, z)) \tilde{N}(d s, d z)\right\}
\end{aligned}
$$

where $\nu$ is the Lévy measure and $\tilde{N}$ is the compensated jump measure on the set $\{z:|z|<R\}$.

We shall see how the Product Formula can be used to prove a generalized version of this expression with respect to a full (as opposed to a pure jump) Lévy process. To look at geometric Lévy processes from a nonstandard perspective, let $L$ be a (one-dimensional) hyperfinite Lévy process and let $B$ be an Anderson random walk (this is just a Bernoulli random walk on $T$ with stepsize $\pm \sqrt{\Delta t}$, see, e.g., [1, page 78]) independent of $L$, and consider a stochastic difference equations of the form

$$
\Delta X_{t}=X_{t}\left(\alpha \Delta t+\beta \Delta B_{t}+\Gamma\left(\omega, \Delta L_{t}, t\right)\right)
$$

where $\Gamma(\omega, 0, t)=0$. By induction, the solution to this equation is

$$
X_{t}=X_{0} \prod_{s=0}^{t}\left(1+\alpha \Delta t+\beta \Delta B_{s}+\Gamma\left(\omega, \Delta L_{s}, s\right)\right)
$$

We shall apply the Product Formula to the augmented process

$$
\tilde{L}(\omega, t):=\left(t, B_{t}, L(t)\right)
$$

and the function $\psi: \Omega \times{ }^{*} \mathbf{R}^{3} \times T \rightarrow{ }^{*} \mathbf{R}$ defined by

$$
\psi(\omega, x, y, z, t)=1+\alpha x+\beta y+\Gamma(\omega, z, t)
$$

Before we begin, observe that the the covariance matrix $C^{\eta}$ of $\tilde{L}$ takes the form

$$
C^{\eta} \approx\left(\begin{array}{ccc}
0 & 0 & 0 \\
0 & 1 & 0 \\
0 & 0 & c^{2}
\end{array}\right)
$$

for a constant $c$ ( $c$ is the diffusion coefficient of $L$ ). We also observe that

$$
\begin{aligned}
& \prod_{s=0}^{t} \psi\left(\omega, \Delta \tilde{L}^{>\eta}(s), s\right) e^{-\nabla \psi(\omega, 0, s) \cdot \Delta \tilde{L}^{>\eta}(s)} \approx \\
& \approx \prod_{s=0}^{t}\left(1+\Gamma\left(\omega, \Delta L^{>\eta}(s), s\right)\right) e^{-\Gamma_{z}(\omega, 0, s) \Delta L_{s}^{>\eta}}
\end{aligned}
$$


where $\Gamma_{z}(\omega, 0, s)=\frac{\partial \Gamma}{\partial z}(\omega, 0, s)$. The Product Formula now gives

$$
\begin{gathered}
X_{t}=X_{0} \prod_{s=0}^{t}\left(1+\alpha \Delta t+\beta \Delta B_{s}+\Gamma\left(\omega, \Delta L_{s}, s\right)\right)=X_{0} \prod_{s=0}^{t} \psi\left(\omega, \Delta \tilde{L}_{s}, s\right) \approx \\
\approx X_{0}\left[\prod_{s=0}^{t}\left(1+\Gamma\left(\omega, \Delta L^{>\eta}(s), s\right)\right) e^{-\Gamma_{z}(\omega, 0, s) \Delta L_{s}^{>\eta}}\right] \times \\
\times \exp \left(\left(\alpha-\frac{\beta^{2}}{2}\right) t+\beta B_{t}+\int_{0}^{t} \Gamma_{z}(\omega, 0, s) d L_{s}+\frac{c^{2}}{2} \int_{0}^{t}\left(\Gamma_{z z}-\Gamma_{z}^{2}\right)(\omega, 0, s) d s\right)
\end{gathered}
$$

To compare this formula to the one from [12] above, it is convenient to rewrite the product term

$$
\prod_{s=0}^{t}\left(1+\Gamma\left(\omega, \Delta L^{>\eta}(s), s\right)\right) e^{-\Gamma_{z}(\omega, 0, s) \Delta L_{s}^{>\eta}}
$$

as

$$
e^{\sum_{s=0}^{t}\left\{\ln \left(1+\Gamma\left(\omega, \Delta L^{>\eta}(s), s\right)\right)-\Gamma_{z}(\omega, 0, s) \Delta L_{s}^{>\eta}\right\}}
$$

assuming that $\Gamma>-1$ for the time being. Except for some notational differences, the formulas have a lot in common, but we have an extra term $\frac{c^{2}}{2} \int_{0}^{t}\left(\Gamma_{z z}-\right.$ $\left.\Gamma_{z}^{2}\right)(\omega, 0, s) d s$ coming from the diffusion part of our Lévy process $L$, and there is also a slight difference in the way the two formulas treat the divergence problems of the Lévy measure - we are "normalizing" with the linearized term $-\Gamma_{z}(\omega, 0, s) \Delta L_{s}$ while [12] makes use of the nonlinearized term $-\gamma\left(\omega, \Delta l_{s}, s\right)$. Observe also that as we are using products for the "jump part" of the expression, we do not need the requirement $\gamma \geq-1$ of [12].

\section{Transforming increments}

Since a hyperfinite Lévy process is given in terms of a hyperfinite set $A$ of increments and an internal set $\left\{p_{a}\right\}_{a \in A}$ of transition probabilities, there are two natural ways to transform it into another hyperfinite Lévy process - we can either change the increments, or we can change the transition probabilities. In this section we shall study what happens when we change the increments, and in the next we shall take a look at what happens when we change the transition probabilities.

Assume that $\phi:{ }^{*} \mathbf{R}^{d} \rightarrow{ }^{*} \mathbf{R}^{m}$ is an internal function and consider a hyperfinite Lévy process $L$ with increments $a \in A$ and transition probabilities $\left\{p_{a}\right\}_{a \in A}$. We define a new hyperfinite random walk ${ }^{\phi} L$ in ${ }^{*} \mathbf{R}^{m}$ by

$$
{ }^{\phi} L(\omega, t)=\sum_{s=0}^{t} \phi(\Delta L(\omega, s))
$$


This is obviously a hyperfinite random walk with increments ${ }^{\phi} A:=\{\phi(a)$ : $a \in A\}$ and transition probabilities $\left\{p_{a}\right\}_{a \in A}$ (to be perfectly consistent in our notation, we should rename this set $\left\{p_{b}\right\}_{b \in \phi}$, but that would just be confusing). The function $\phi$ should map infinitesimals to infinitesimals, and there is no great loss of generality to assume that $\phi(0)=0$ (if not, we just adjust $\phi$ by an infinitesimal). We want to know when ${ }^{\phi} L$ is a hyperfinite Lévy process (and not just a hyperfinite random walk), and the following simple lemma gives us a useful criterion. Recall the definition of $\mathcal{S C}^{2}\left({ }^{*} \mathbf{R}^{d},{ }^{*} \mathbf{R}^{m}\right)$ from the beginning of Section 3 .

Lemma 6.1 If $L$ is a hyperfinite Lévy process and $\phi \in \mathcal{S C}^{2}\left({ }^{*} \mathbf{R}^{d},{ }^{*} \mathbf{R}^{m}\right)$ with $\phi(0)=0$, then ${ }^{\phi} L$ is a hyperfinite Lévy process.

Proof: Assume first that $L$ has finite increments. Then ${ }^{\phi} L$ has finite increments, and according to Corollary 1.5 we only have to prove that:

(i) $\frac{1}{\Delta t} \sum_{a \in A} \phi(a) p_{a}$ is finite

(ii) $\frac{1}{\Delta t} \sum_{a \in A}|\phi(a)|^{2} p_{a}$ is finite

To prove (i), observe that by Taylor's formula

$$
\phi(a)=\nabla \phi(0) \cdot a+\frac{1}{2} \sum_{i, j} \frac{\partial^{2} \phi}{\partial x_{i} \partial x_{j}}\left(\theta_{a}\right) a_{i} a_{j}
$$

for some $\theta_{a}$ on the line segment from 0 to $a$. Since the increments $a$ are bounded by a real number and $\phi \in \mathcal{S C}^{2}\left({ }^{*} \mathbf{R}^{d},{ }^{*} \mathbf{R}^{m}\right)$, we have $\frac{1}{2} \sum_{i, j} \frac{\partial^{2} \phi}{\partial x_{i} \partial x_{j}}\left(\theta_{a}\right) a_{i} a_{j} \leq$ $K|a|^{2}$ for some real number $K$. Hence

$$
\frac{1}{\Delta t}\left|\sum_{a \in A} \phi(a) p_{a}\right| \leq \frac{|\nabla \phi(0)|}{\Delta t} \cdot\left|\sum_{a \in A} a p_{a}\right|+\frac{K}{\Delta t} \sum_{a \in A}|a|^{2} p_{a}
$$

which is finite since $L$ is a hyperfinite Lévy process with finite increments.

The proof of (ii) is similar, but easier. This time we just need the first order Taylor approximation

$$
\phi(a)=\nabla \phi\left(\theta_{a}\right) \cdot a
$$

for a $\theta_{a}$ on the line segment from 0 to $a$. Since $\left\{\nabla \phi\left(\theta_{a}\right)\right\}_{a \in A}$ is bounded by a real constant $K$, we have:

$$
\frac{1}{\Delta t} \sum_{a \in A}|\phi(a)|^{2} p_{a} \leq \frac{K^{2}}{\Delta t} \sum_{a \in A}|a|^{2} p_{a}
$$

which is finite since $L$ is a hyperfinite Lévy process with finite increments.

To extend the result to hyperfinite Lévy processes with infinite increments, just observe that we already proved that ${ }^{\phi}\left(L^{\leq k}\right)$ is a hyperfinite Lévy process 
for all noninfinitesimal, finite $k$. The result follows from Proposition 1.9.

We may now use the Sum Formula 3.5 to find an approximate expression for ${ }^{\phi} L$ :

Proposition 6.2 Let $L$ be a hyperfinite Lévy process and assume that $\phi \in$ $\mathcal{S C}^{2}\left({ }^{*} \mathbf{R}^{d},{ }^{*} \mathbf{R}^{m}\right)$ with $\phi(0)=0$. Then for $P_{L^{-}}$a.a $\omega$ and all finite $t \in T$ :

$$
\begin{gathered}
{ }^{\phi} L(\omega, t) \approx \nabla \phi(0) \cdot L(\omega, t)+\frac{t}{2} \sum_{i, j} C_{i, j}^{\eta} \frac{\partial^{2} \phi}{\partial x_{i} \partial x_{j}}(0)+ \\
+\sum_{s=0}^{t}\left\{\phi\left(\Delta L^{>\eta}(\omega, s)\right)-\nabla \phi(0) \cdot \Delta L^{>\eta}(\omega, s)\right\}
\end{gathered}
$$

where $\eta$ is any splitting infinitesimal for $L$, and $C^{\eta}=\left\{C_{i, j}^{\eta}\right\}$ is the corresponding infinitesimal covariance matrix.

Proof: This is just a special case of the Sum Formula 3.5.

\section{Transforming probabilities}

In this section, we keep the increments $a \in A$ of our hyperfinite Lévy process $L$, but change the transition probabilities from $\left\{p_{a}\right\}_{a \in A}$ to $\left\{q_{a}\right\}_{a \in A}$ where $q_{a}=$ $\psi(a) p_{a}$ for some function $\psi:{ }^{*} \mathbf{R}^{d} \rightarrow^{*}[0, \infty)$. We obviously need

$$
\sum_{a \in A} \psi(a) p_{a}=1
$$

to get a probability measure. We shall write $Q$ for the new, induced probability measure on $\Omega$.

If we restrict overselves to a bounded timeline $T_{t}=\{s \in T: s<t\}$ (where $t$ is finite), the density $D$ of the new measure $Q$ with respect to the old measure $P$ is clearly given by

$$
D(\omega, t)=\prod_{s=0}^{t} \psi(\Delta L(\omega, s))
$$

We shall use the Product Formula 4.1 to find an expression for $D$. However, such an expression is of little value unless we know that $Q$ is absolutely continuous with respect to $P$ (or, more correctly, that the Loeb measure $Q_{L}$ of $Q$ is absolutely continuous with respect to the Loeb measure $P_{L}$ of $P$ - at least as long as we restrict to bounded time intervals). Therefore, most of our efforts in this section will go into showing that under quite general conditions, $Q_{L}$ is absolutely continuous with respect to $P_{L}$. Note that when this is the case, our process $L$ is a hyperfinite Lévy process also with respect to the new measure $Q$ (this follows immediately from Definition 1.3). 
We shall be working with two different classes $\mathcal{D}^{1}(L)$ and $\mathcal{D}^{2}(L)$ of functions $\psi$ according to how much differentiability we need (recall the definition of $\mathcal{S C}^{r}\left({ }^{*} \mathbf{R}^{d},{ }^{*} \mathbf{R}^{d}\right)$ from the beginning of Section 3$)$ :

Definition 7.1 Let $L$ be a hyperfinite Lévy process with transition probabilities $\left\{p_{a}\right\}_{a \in A}$. We define $\mathcal{D}^{r}(L)$ (where $r \in \mathbf{N}$ ) to be the set of all internal functions $\psi:{ }^{*} \mathbf{R}^{d} \rightarrow^{*}[0, \infty)$ such that:

(i) $\psi \in \mathcal{S C}^{r}\left({ }^{*} \mathbf{R}^{d},{ }^{*}[0, \infty)\right)$

(ii) $\sum_{a \in A} \psi(a) p_{a}=1$

(iii) $\lim _{k \rightarrow \infty} \circ\left(\frac{1}{\Delta t} \sum_{|a|>k} \psi(a) p_{a}\right)=0$

We begin with a simple lemma which will allow us to reduce many arguments to processes with finite increments.

Lemma 7.2 Let $L$ be a hyperfinite Lévy process and assume that $\psi \in \mathcal{D}^{r}(L)$ for some $r \in \mathbf{N}$. Then there exist finite numbers $k, c$ such that the modified function $\tilde{\psi}(a)=\psi(a) /(1-c \Delta t)$ is in $\mathcal{D}^{r}\left(L^{<k}\right)$.

Proof: By part (iii) in the definition of $\mathcal{D}^{r}(L)$, there is a finite (and noninfinitesimal) $k$ such that $\sum_{|a|<k} \psi(a) p_{a}=1-m \Delta t$ for some finite $m$. From the general theory of hyperfinite Lévy processes, we know that $\sum_{\{a \in A: a \geq k\}} p_{a}=n \Delta t$ for some finite $n$. If $A^{<k}$ is the set of increments of the truncated process $L^{<k}$, then clearly, $A^{<k}=\{0\} \cup\{a \in A:|a|<k\}$. Hence

$\sum_{a \in A^{<k}} \psi(a) p_{a}=\sum_{|a|<k} \psi(a) p_{a}+\sum_{|a| \geq k} \psi(0) p_{a}=(1-m \Delta t)+\psi(0) n \Delta t=1-c \Delta t$

where $c=m-\psi(0) n$. This means that $\tilde{\psi}(a)=\psi(a) /(1-c \Delta t)$ satisfies part (ii) in the definition of $\mathcal{D}^{r}(L)$, and the other two conditions are trivially satisfied.

The next two lemmas show that the classes $\mathcal{D}^{r}$ have more structure than may be obvious at first glance.

Lemma 7.3 Let $L$ be a hyperfinite Lévy process.

(i) If $\psi \in \mathcal{D}^{2}(L)$, then $\psi(0)=1+\lambda \Delta$ t for some finite $\lambda$.

(ii) If $\psi \in \mathcal{D}^{1}(L)$, then $\psi(0)=1+\gamma \sqrt{\Delta t}$ for some finite $\gamma$.

Proof: By the previous lemma we may assume that $L$ has finite increments.

(i) By Taylor's formula: 


$$
\begin{gathered}
1=\sum_{a \in A} \psi(a) p_{a}=\sum_{a \in A} \psi(0) p_{a}+\sum_{a \in A}(\psi(a)-\psi(0)) p_{a}= \\
=\psi(0)+\sum_{a \in A} \nabla \psi(0) \cdot a p_{a}+\frac{1}{2} \sum_{a \in A} \sum_{i, j} \frac{\partial^{2} \psi}{\partial x_{i} \partial x_{j}}\left(\theta_{a}\right) a_{i} a_{j} p_{a}= \\
=\psi(0)+\nabla \psi(0) \cdot \mu_{L} \Delta t+\frac{1}{2} \sum_{a \in A} \sum_{i, j} \frac{\partial^{2} \psi}{\partial x_{i} \partial x_{j}}\left(\theta_{a}\right) a_{i} a_{j} p_{a}
\end{gathered}
$$

for some $\theta_{a}$ between 0 and $a$. Since $L$ has finite increments, $\frac{1}{2} \frac{\partial^{2} \psi}{\partial x_{i} \partial x_{j}}\left(\theta_{a}\right)$ is bounded by a finite number $C$, and hence

$$
\frac{1}{2} \sum_{a \in A} \sum_{i, j} \frac{\partial^{2} \psi}{\partial x_{i} \partial x_{j}}\left(\theta_{a}\right) a_{i} a_{j} p_{a} \leq \sum_{a \in A} \sum_{i, j} C|a|^{2} p_{a} \leq C \sigma_{L}^{2} \Delta t
$$

and the result follows.

(ii) We use essentially the same argument, but have one less derivative to play with:

$1=\sum_{a \in A} \psi(a) p_{a}=\sum_{a \in A} \psi(0) p_{a}+\sum_{a \in A}(\psi(a)-\psi(0)) p_{a}=\psi(0)+\sum_{a \in A} \nabla \psi\left(\theta_{a}\right) \cdot a p_{a}$

Since $L$ has finite increments, $\left|\nabla \psi\left(\theta_{a}\right)\right|$ is bounded by a constant $K$, and hence by Hölder's inequality:

$$
\begin{gathered}
\sum_{a \in A} \nabla \psi\left(\theta_{a}\right) \cdot a p_{a} \leq K \sum_{a \in A}|a| p_{a} \leq K\left(\sum_{a \in A}|a|^{2} p_{a}\right)^{\frac{1}{2}}\left(\sum_{a \in A}|1|^{2} p_{a}\right)^{\frac{1}{2}}= \\
=\left(\sum_{a \in A}|a|^{2} p_{a}\right)^{\frac{1}{2}}=\sqrt{\Delta t} \sigma_{L}
\end{gathered}
$$

The result follows.

Lemma 7.4 Let $L$ be a hyperfinite Lévy process and assume that $\psi \in \mathcal{D}^{1}(L)$. Then there is a finite number $\xi$ such that $\sum_{a \in A} \psi(a)^{2} p_{a}=1+\xi \Delta t$

Proof: By Lemma 7.2 we need only consider processes with finite increments. Observe first that since

$$
\xi:=\frac{1}{\Delta t} \sum_{a \in A}(\psi(a)-1)^{2} p_{a}=\frac{1}{\Delta t} \sum_{a \in A}\left(\psi(a)^{2}-2 \psi(a)+1\right) p_{a}=
$$




$$
=\frac{1}{\Delta t}\left(\sum_{a \in A} \psi(a)^{2} p_{a}-1\right)
$$

it suffices to show that $\frac{1}{\Delta t} \sum_{a \in A}(\psi(a)-1)^{2} p_{a}$ is finite. We have

$$
\begin{gathered}
\frac{1}{\Delta t} \sum_{a \in A}(\psi(a)-1)^{2} p_{a}=\frac{1}{\Delta t} \sum_{a \in A}(\psi(a)-\psi(0)+\psi(0)-1)^{2} p_{a} \leq \\
\leq \frac{2}{\Delta t} \sum_{a \in A}(\psi(a)-\psi(0))^{2} p_{a}+\frac{2}{\Delta t} \sum_{a \in A}(\psi(0)-1)^{2} p_{a}
\end{gathered}
$$

The last term is finite by the previous lemma, and the first is finite by yet another exercise in Taylor's formula:

$$
\frac{2}{\Delta t} \sum_{a \in A}(\psi(a)-\psi(0))^{2} p_{a} \leq \frac{2}{\Delta t} \sum_{a \in A}\left|\nabla \psi\left(\theta_{a}\right)\right|^{2}|a|^{2} p_{a} \leq M \sigma_{L}^{2}
$$

where $M$ is a finite number bounding $2\left|\nabla \psi\left(\theta_{a}\right)\right|^{2}$.

We are now ready to show that the density process $D_{t}(\omega)=\prod_{s=0}^{t} \psi(\Delta L(\omega, s))$ is S-integrable. This implies that the new Loeb measure $Q_{L}$ is absolutely continuous with respect to the old $P_{L}$ on bounded intervals.

Proposition 7.5 Let $L$ be a hyperfinite Lévy process and assume that $\psi \in$ $\mathcal{D}^{1}(L)$. Then $D_{t}(\omega)=\prod_{s=0}^{t} \psi(\Delta L(\omega, s))$ is S-integrable for all finite $t \in T$.

Proof: It suffices to show that $E\left(D(t)^{2}\right)$ is finite. By Lemma 7.4

$$
\begin{aligned}
& E\left(D(t)^{2}\right)=E\left(\prod_{s=0}^{t} \psi(\Delta L(s))^{2}\right)=\prod_{s=0}^{t} E\left(\psi(\Delta L(s))^{2}\right)= \\
= & \prod_{s=0}^{t} \sum_{a \in A} \psi(a)^{2} p_{a}=\prod_{s=0}^{t}(1+\xi \Delta t)=(1+\xi \Delta t)^{\frac{t}{\Delta t}} \approx e^{\xi t}
\end{aligned}
$$

which is finite.

Finally, we use the Product Formula 4.1 to find an expression for the density process $D$ :

Theorem 7.6 Let $L$ be a hyperfinite Lévy process and assume that $\psi \in \mathcal{D}^{2}(L)$. Then for all finite $t \in T$, the product $D_{t}(\omega)=\prod_{s=0}^{t} \psi(\Delta L(\omega, s))$ is S-integrable and

$$
\begin{aligned}
& D_{t}(\omega) \approx\left(\prod_{s=0}^{t} \psi\left(\Delta L^{>\eta}(\omega, s)\right) e^{-\nabla \psi(0) \cdot \Delta L^{>\eta}(\omega, s)}\right) \times \\
& \times e^{\lambda t+\nabla \psi(0) \cdot L(\omega, t)+\frac{t}{2} \sum_{i, j} C_{i, j}^{\eta}\left[\frac{\partial^{2} \psi}{\partial x_{i} \partial x_{j}}(0)-\frac{\partial \psi}{\partial x_{i}}(0) \frac{\partial \psi}{\partial x_{j}}(0)\right]}
\end{aligned}
$$

where $\eta$ is a splitting infinitesimal, $C^{\eta}$ the corresponding infinitesimal covariance matrix, and $\lambda=(\psi(0)-1) / \Delta t$ is finite. 
Proof: According to Lemma 7.3, $\psi(0)=1+\lambda \Delta t$ for a finite constant $\lambda$. Applying the Product Formula 4.1 to the function $\tilde{\psi}(x)=\frac{\psi(x)}{1+\lambda \Delta t}$ and observing that $(1+\lambda \Delta t)^{t / \Delta t} \approx e^{\lambda t}$, we get the formula. The S-integrability is already established in the proposition above.

\section{Minimal martingale measures}

Let $L$ be a $d$-dimensional hyperfinite Lévy process and assume that $\phi_{i} \in N I(L)$ for $i=1, \ldots, d$. Consider the $d$-dimensional, nonlinear stochastic integral

$$
X(\omega, t)=\int_{0}^{t} \phi\left(\omega, d L_{s}(\omega), s\right)
$$

defined componentwise by

$$
X_{i}(\omega, t)=\int_{0}^{t} \phi_{i}\left(\omega, d L_{s}(\omega), s\right)
$$

We want to change the probability measure $P$ into a new measure $Q$ such that $X$ is a martingale with respect to $Q$ and such that the Loeb measure $Q_{L}$ of $Q$ is absolutely continuous with respect to the Loeb measure $P_{L}$ of $P-$ at least as long as we restrict our processes to bounded time intervals.

The increments of $X$ at time $t$ are $\phi(\omega, a, t)$ where $a \in A$. If $X$ is to be a martingale, we must give the increments a distribution $a \mapsto q(\omega, a, t)$ (which now depends on time and history) such that

$$
\sum_{a \in A} \phi(\omega, a, t) q(\omega, a, t)=0
$$

It is not always possible to find a new measure $q$ which turns $X$ into a martingale - e.g., no change of measure can make a martingale out of a strictly increasing process. In fact, the formula above tells us that it is possible to turn $X$ into a martingale by a change of measure if and only if for all $\omega$ and $t$, the origin is in the convex hull of the set $\{\phi(\omega, a, t): a \in A\}$ of increments (and in that case there are often many possibilities corresponding to different convex combinations). This is one of the fundamental observations of discrete time mathematical finance, but it is of little use in the present setting as it may produce measures $Q$ which are hopelessly singular with respect to $P$.

For a more realistic approach, we assume that $q$ is given by a density $\psi$, i.e., $q(\omega, a, t)=\psi(\omega, a, t) p_{a}$. The formula above then becomes

$$
\sum_{a \in A} \phi(\omega, a, t) \psi(\omega, a, t) p_{a}=0
$$

and in addition we have 


$$
\sum_{a \in A} \psi(\omega, a, t) p_{a}=1
$$

since $q$ is a probability measure. We also need, of course, that $\psi(\omega, a, t) \geq 0$.

If we can can find such a function $\psi$, our process $X$ will be a martingale with respect to the new measure $Q$ on $\Omega$ defined by

$$
Q(\omega)=\prod_{s \in T} \psi(\omega, \Delta L(\omega, s), s) p_{\Delta L(\omega, s)}
$$

The density of $Q$ with respect to the original measure $P$ is given by the process

$$
D_{t}(\omega)=\prod_{s=0}^{t} \psi(\omega, \Delta L(\omega, s), s)
$$

and we hope to use the Product Formula to find an approximate expression for this process. However, for such a formula to be of much use, we need the new measure $Q$ to be absolutely continuous with respect to the old. Even with this condition satisfied, there are often several candidates for $Q$ to choose among (known by confusingly similar names such as the minimal martingale measure, the minimal variation martingale measure, and - several versions of - the minimal entropy martingale measure). We shall concentrate here on the notion of a minimal martingale measure introduced by Föllmer and Schweizer in [5] (see [14], [15], [4] for more information, and consult also [10] for an efficient way to find martingale measures) as it is the algebraically simplest, but it should be possible to do similar calculations for the other candidates.

The idea behind the minimal martingale measure is that we want a measure which turns $\int \phi\left(\omega, d L_{t}, t\right)$ into a martingale, but which preserves as many other martingales as possible. Let, as usual, $\left\{\mathcal{F}_{t}\right\}$ be the internal filtration generated by $L$. An $\left\{\mathcal{F}_{t}\right\}$-martingale $M: \Omega \times T \rightarrow^{*} \mathbf{R}$ is orthogonal to $L$ if

$$
E\left[\Delta M(t) \Delta L_{i}(t) \mid \mathcal{F}_{t}\right]=0 \quad \text { for all } t \in T \text { and all } i=1, \ldots, d
$$

Here is our adaption of Föllmer's and Schweizer's concept of a minimal martingale measure:

Definition 8.1 Let $\psi: \Omega \times^{*} \mathbf{R}^{d} \times T \rightarrow^{*}[0, \infty)$ be a nonanticipating function such that $\sum_{a \in A} \psi(\omega, a, t) p_{a}=1$ for all $\omega$ and $t$, and let

$$
Q(\omega)=\prod_{t \in T} \psi(\omega, \Delta L(\omega, t), t) p_{\Delta L(\omega, t)}
$$

be the internal probability measure on $\Omega$ induced by $\psi$. Consider the following conditions:

(i) any internal martingale (w.r.t. $P$ ) which is orthogonal to $L$ is also a martingale with respect to $Q$. 
(ii) $\int \phi\left(\omega, d L_{t}, t\right)$ is a martingale with respect to $Q$.

(iii) the density $D_{t}(\omega)=\prod_{s=0}^{t} \psi(\omega, \Delta L(\omega, s), s)$ is S-integrable for all finite $t$.

If condition (i) is satisfied, we call $Q$ a minimal measure with respect to $L$. If in addition (ii) is satisfied, we call $Q$ a minimal martingale measure for $\int \phi\left(\omega, d L_{t}, t\right)$ with respect to $L$. If all three conditions are satisfied, we call $Q$ an absolutely continuous minimal martingale measure for $\int \phi\left(\omega, d L_{t}, t\right)$ with respect to $L$.

In our hyperfinite setting, it is just an exercise in linear algebra to show that a minimal martingale measure is unique if it exists. To find candidates for $Q$, we look at density functions $\psi$ which are affine in $a$ in the sense that

$$
\psi(\omega, a, t)=\alpha(\omega, t)+\sum_{j=1}^{d} \beta_{j}(\omega, t) a_{j}
$$

where $\alpha, \beta_{1}, \ldots, \beta_{d}$ are nonanticipating processes taking values in ${ }^{*} \mathbf{R}$ and $a=$ $\left(a_{1}, \ldots, a_{d}\right) \in{ }^{*} \mathbf{R}^{d}$. An easy computation shows that such affine processes generate minimal measures (if they generate measures at all!):

Lemma 8.2 Assume that $\alpha, \beta_{1}, \ldots, \beta_{d}$ are nonanticipating processes taking values in ${ }^{*} \mathbf{R}$ and let $\psi(\omega, a, t)=\alpha(\omega, t)+\sum_{j=1}^{d} \beta_{j}(\omega, t) a_{j}$. Assume further that $\psi(\omega, a, t) \geq 0$ for all $\omega, a, t$ and that $\sum_{a \in A} \psi(\omega, a, t) p_{a}=1$ for all $\omega, t$. Then the measure $Q$ on $\Omega$ generated by $\psi$ is a minimal measure for $L$.

Proof: Assume that $M$ is a martingale orthogonal to $L$. Then

$$
\begin{gathered}
E_{Q}\left[\Delta M(t) \mid \mathcal{F}_{t}\right]=E_{P}\left[\Delta M(t) \prod_{s \in T}\left\{\alpha(s)+\sum_{j=1}^{d} \beta_{j}(s) \Delta L_{j}(s)\right\} \mid \mathcal{F}_{t}\right]= \\
=\prod_{s=0}^{t}\left\{\alpha(s)+\sum_{j=1}^{d} \beta_{j}(s) \Delta L_{j}(s)\right\} E_{P}\left[\Delta M(t)\left\{\alpha(t)+\sum_{j=1}^{d} \beta_{j}(t) \Delta L_{j}(t)\right\} \mid \mathcal{F}_{t}\right]=0
\end{gathered}
$$

where we use the orthogonality in the last step.

To get a minimal martingale measure, we must choose $\alpha, \beta_{1}, \ldots, \beta_{d}$ such that $\int \phi(\omega, \Delta L(t), t)$ is a martingale w.r.t $Q$. If we write (7) componentwise, we get for $i=1, \ldots, d$

$$
0=\sum_{a \in A} \phi_{i}(\omega, a, t) \psi(\omega, a, t) p_{a}=\sum_{a \in A} \phi_{i}(\omega, a, t)\left\{\alpha(\omega, t)+\sum_{j=1}^{d} \beta_{j}(\omega, t) a_{j}\right\} p_{a}=
$$




$$
=\alpha(\omega, t) \sum_{a \in A} \phi_{i}(\omega, a, t) p_{a}+\sum_{j=1}^{d} \beta_{j}(\omega, t) \sum_{a \in A} \phi_{i}(\omega, a, t) a_{j} p_{a}
$$

We may think of this as $d$ equations in the $d+1$ unknowns $\alpha, \beta_{1}, \ldots, \beta_{d}$. To get the last equation, we note that (8) can be written

$$
1=\sum_{a \in A} \psi(\omega, a, t) p_{a}=\sum_{a \in A}\left\{\alpha(\omega, t)+\sum_{j=1}^{d} \beta_{j}(\omega, t) a_{j}\right\} p_{a}=\alpha(\omega, t)+\beta(\omega, t) \cdot \mu \Delta t
$$

where $\mu:=\mu_{L}=\frac{1}{\Delta t} \sum_{a \in A} a p_{a}$ and we think of $\beta(\omega, t)$ as a vector valued process $\beta(\omega, t)=\left(\beta_{1}(\omega, t), \ldots, \beta_{d}(\omega, t)\right)$.

To simplify notation, we introduce

$$
\rho_{i}(\omega, t)=\frac{1}{\Delta t} \sum_{a \in A} \phi_{i}(\omega, a, t) p_{a}
$$

and

$$
M_{i, j}(\omega, t)=\frac{1}{\Delta t} \sum_{a \in A} \phi_{i}(\omega, a, t) a_{j} p_{a}
$$

Assuming that $L$ has finite increments, the usual Taylor arguments show that $\rho_{i}$ and $M_{i, j}$ are finite $P_{L}$-a.e. With this notation, the equations above can be written in matrix form in this way:

$$
\left(\begin{array}{cccc}
1 & \mu_{1} \Delta t & \ldots & \mu_{d} \Delta t \\
\rho_{1} \Delta t & M_{1,1} \Delta t & \ldots & M_{1, d} \Delta t \\
\rho_{2} \Delta t & M_{2,1} \Delta t & \ldots & M_{2, d} \Delta t \\
\vdots & \vdots & \vdots & \vdots \\
\rho_{d} \Delta t & M_{d, 1} \Delta t & \ldots & M_{d, d} \Delta t
\end{array}\right)\left(\begin{array}{c}
\alpha \\
\beta_{1} \\
\beta_{2} \\
\vdots \\
\beta_{d}
\end{array}\right)=\left(\begin{array}{c}
1 \\
0 \\
0 \\
\vdots \\
0
\end{array}\right)
$$

where we have suppressed the dependence on $\omega$ and $t$ to increase readability. We shall assume that the matrix $M=\left\{M_{i, j}\right\}$ is uniformly nonsingular in the sense that it has finite entries and that for all finite $t$, there exists an $\epsilon_{t} \in \mathbf{R}_{+}$ such that $\operatorname{det}(M)(\omega, s) \geq \epsilon_{t}$ for all $\omega$ and all $s \leq t$. Let $\tilde{\beta}=\left(\tilde{\beta}_{1}, \ldots, \tilde{\beta}_{d}\right)$ be the solution of

$$
\left(\begin{array}{ccc}
M_{1,1} & \ldots & M_{1, d} \\
M_{2,1} & \ldots & M_{2, d} \\
\vdots & \vdots & \vdots \\
M_{d, 1} & \ldots & M_{d, d}
\end{array}\right)\left(\begin{array}{c}
\tilde{\beta}_{1} \\
\tilde{\beta}_{2} \\
\vdots \\
\tilde{\beta}_{d}
\end{array}\right)=\left(\begin{array}{c}
-\rho_{1} \\
-\rho_{2} \\
\vdots \\
-\rho_{d}
\end{array}\right)
$$

We now introduce new variables $x, y_{1}, \ldots, y_{n}$ by $\left(\alpha, \beta_{1}, \ldots, \beta_{d}\right)=\left(x, y_{1}, \ldots, y_{d}\right)+$ $\left(1, \tilde{\beta}_{1}, \ldots, \tilde{\beta}_{d}\right)$. The system (9) then becomes

$$
\left(\begin{array}{cccc}
1 & \mu_{1} \Delta t & \ldots & \mu_{d} \Delta t \\
\rho_{1} \Delta t & M_{1,1} \Delta t & \ldots & M_{1, d} \Delta t \\
\rho_{2} \Delta t & M_{2,1} \Delta t & \ldots & M_{2, d} \Delta t \\
\vdots & \vdots & \vdots & \vdots \\
\rho_{d} \Delta t & M_{d, 1} \Delta t & \ldots & M_{d, d} \Delta t
\end{array}\right)\left(\begin{array}{c}
x \\
y_{1} \\
y_{2} \\
\vdots \\
y_{d}
\end{array}\right)=\left(\begin{array}{c}
-(\tilde{\beta} \cdot \mu) \Delta t \\
0 \\
0 \\
\vdots \\
0
\end{array}\right)
$$


Using Cramer's rule, it is easy to check that this system has a unique solution where all entries are of order of magnitude $\Delta t$. By Cramer's rule, we also see that $x \approx-\tilde{\beta} \cdot \mu_{L} \Delta t$ with an error that is infinitesimal compared to $\Delta t$.

There is one condition we have not taken into account yet — we need $q$ to be positive, i.e., we need $\sum_{i=1}^{d} \beta_{i}(\omega, t) a_{i} \geq-\alpha(\omega, t)$ for all $\omega, a, t$. For processes with jumps, this condition is quite restrictive, but it is the price we have to pay for working with affine functions $\psi$ (i.e. with minimal martingale measures). Note that if we allow signed measures $Q$ (which technically works well), the problem disappears. Note also that since $\alpha(\omega, t) \approx 1$ and $\beta_{i} \approx \tilde{\beta}_{i}$, the condition is satisfied if $\sum_{i=1}^{d} \tilde{\beta}_{i}(\omega, t) a_{i} \gtrsim-1$ for all $a, \omega, t$.

We now have a minimal martingale measure $Q$ which we want to show is absolutely continuous on bounded intervals.

Lemma 8.3 Let $L$ be a hyperfinite Lévy process with finite increments, and assume that $\gamma, \beta_{1}, \ldots, \beta_{d}$ are nonanticipating, S-bounded processes. Then the process

$$
D_{t}(\omega)=\prod_{s=0}^{t}\left(1+\gamma(\omega, s) \Delta t+\sum_{i=1}^{d} \beta_{i}(\omega, s) \Delta L_{i}(\omega, s)\right)
$$

is $S$-integrable for all finite $t$.

Proof: It suffices to prove that $E\left(D(t)^{2}\right)$ is finite for all finite $t$. Observe that

$$
\begin{gathered}
E\left[D(t+\Delta t)^{2}\right]=E\left[D(t)^{2}\left(1+\gamma(t) \Delta t+\sum_{i=1}^{d} \beta_{i}(t) \Delta L_{i}(t)\right)^{2}\right]= \\
=E\left[D(t)^{2}(1+\gamma(t) \Delta t)^{2}\right]+2 E\left[D(t)^{2}(1+\gamma(t) \Delta t) \sum_{i=1}^{d} \beta_{i}(t) E\left[\Delta L_{i}(t) \mid \mathcal{F}_{t}\right]\right]+ \\
+E\left[D(t)^{2} \sum_{i, j}^{d} \beta_{i}(t) \beta_{j}(t) E\left[\Delta L_{i}(t) \Delta L_{j}(t) \mid \mathcal{F}_{t}\right]\right]
\end{gathered}
$$

If $K$ is a finite number which bounds $|\gamma|,\left|\beta_{1}\right|, \ldots,\left|\beta_{d}\right|$, we see that the first term on the right is bounded by $E\left[D(t)^{2}\right](1+K \Delta t)^{2}<E\left[D(t)^{2}\right](1+3 K \Delta t)$. Since $E\left[\Delta L_{i}(t) \mid \mathcal{F}_{t}\right]=\mu_{i} \Delta t$, the second term is bounded by $3 E\left[D(t)^{2}\right] d K\left|\mu_{L}\right| \Delta t$, and since $E\left[\Delta L_{i}(t) \Delta L_{j}(t) \mid \mathcal{F}_{t}\right]=C_{i, j}^{L} \Delta t \leq \sigma_{L}^{2} \Delta t$, the third term is less than $E\left[D(t)^{2}\right] d^{2} K^{2} \sigma_{L}^{2} \Delta t$ (see the remark at the end of Section 2). Combining these estimates, we see that there is a finite $M$ independent of $t$ such that

$$
E\left[D(t+\Delta t)^{2}\right] \leq E\left[D(t)^{2}\right](1+M \Delta t)
$$

By induction, we have

$$
E\left[D(t)^{2}\right] \leq(1+M \Delta t)^{\frac{t}{\Delta t}} \approx e^{M t}
$$

which is finite.

We are now ready for the main theorem: 
Theorem 8.4 Let $L$ be a hyperfinite Lévy process with finite increments. Assume that $\phi_{i} \in N I(L)$ for $i=1, \ldots, d$, and let

$$
X(\omega, t)=\int_{0}^{t} \phi\left(\omega, d L_{s}(\omega), s\right)
$$

be a multidimensional, nonlinear stochastic integral. Assume that the vector $\rho(\omega, t)$ and the matrix $M(\omega, t)$ are $S$-bounded for all $\omega$ and all finite $t$, and that $M$ is uniformly nonsingular. Assume further that the vector

$$
\tilde{\beta}(\omega, t)=-M(\omega, t)^{-1} \rho(\omega, t)
$$

satisfies $\sum_{i=1}^{d} \tilde{\beta}_{i}(\omega, t) a_{i} \gtrsim-1$ for all $a \in A, \omega \in \Omega$ and all finite $t \in T$. Then there exist nonanticipating, $S$-bounded processes $\alpha, \beta_{1}, \ldots, \beta_{d}$ such that $\alpha-1, \beta_{1}-\tilde{\beta}_{1}, \ldots, \beta_{d}-\tilde{\beta}_{d}$ are of order of magnitude $\Delta t$ for all $\omega$ and all finite $t$, and such that the measure $Q$ generated by $\psi(\omega, a, t)=\alpha(\omega, t)+\sum_{i=1}^{d} \beta_{i}(\omega, t) a_{i}$ is an absolutely continuous minimal martingale measure for $X$ with respect to $L$. The density of $Q$ is given by

$$
D_{t}(\omega) \approx\left(\prod_{s=0}^{t}\left(1+\beta \cdot \Delta L^{>\eta}(\omega, s)\right) e^{-\beta \cdot \Delta L^{>\eta}(\omega, s)}\right) e^{-t \beta \cdot \mu_{L}+\beta \cdot L(\omega, t)-\frac{t}{2}\left\langle C^{\eta} \beta, \beta\right\rangle}
$$

where $\eta$ is a splitting infinitesimal and $C^{\eta}$ the corresponding infinitesimal covariance matrix.

Proof: We have been through most of the argument, and all that remains is some bookkeeping. First note that since $M$ and $\rho$ are S-bounded and $M$ is strictly nonsingular, the vector $\tilde{\beta}$ is finite. Using (11) as above, we see that the solution $\left(\alpha, \beta_{1}, \ldots, \beta_{d}\right)$ differs from $\left(1, \tilde{\beta}_{1}, \ldots, \tilde{\beta}_{d}\right)$ by order of magnitude $\Delta t$, and that $\alpha \approx 1-\left(\tilde{\beta} \cdot \mu_{L}\right) \Delta t$ with an error that is infinitesimal compared to $\Delta t$. By the condition $\sum_{i=1}^{d} \tilde{\beta}_{i}(\omega, t) a_{i} \gtrsim-1$, we get that $\psi(\omega, a, t)=\alpha(\omega, t)+\sum_{i=1}^{d} \beta_{i}(\omega, t) a_{i}$ is positive and hence generates a new measure $Q$ on $\Omega$. By construction, $X$ is a martingale with respect to $Q$, and Lemma 8.2 then tells us that $Q$ is a minimal martingale measure. By the last lemma above, the density $D_{t}$ is S-integrable with respect to $P$, and hence $Q$ is an absolutely continuous minimal martingale measure.

To prove the formula for $D_{t}$, we shall apply the Product Formula 4.1 to the expression $D_{t}(\omega)=\prod_{s=0}^{t} \psi\left(\omega, \Delta L_{s}(\omega), s\right)$, but we need to take a little care as the Product Formula assumes that $\psi(0)=1$, while our $\psi$ only satisfies $\psi(0)=\alpha=1-\left(\tilde{\beta} \cdot \mu_{L}\right) \Delta t+o(\Delta t)$. As in the previous section, the trick is to apply the Product Formula to the function $\hat{\psi}=\psi / \alpha$ and note that $\alpha \frac{t}{\Delta t} \approx e^{-t \beta \cdot \mu_{L}}$. Using that $\hat{\psi}(\omega, a, s)=1+\sum_{j=1}^{d} \hat{\beta}_{j}(\omega, s) a_{j}$ where $\hat{\beta}_{j}(\omega, s):=\beta_{j}(\omega, s) / \alpha(\omega, s) \approx$ $\beta(\omega, s)$, we get:

$$
D(\omega, t)=\prod_{s=0}^{t} \psi\left(\omega, \Delta L_{s}(\omega), s\right)=\alpha^{\frac{t}{\Delta t}} \prod_{s=0}^{t} \hat{\psi}\left(\omega, \Delta L_{s}(\omega), s\right) \approx
$$




$$
\begin{aligned}
& \approx e^{-t \beta \cdot \mu_{L}}\left(\prod_{s=0}^{t}\left(1+\hat{\beta} \cdot \Delta L^{>\eta}(\omega, s)\right) e^{-\hat{\beta} \cdot \Delta L^{>\eta}(\omega, s)}\right) e^{\hat{\beta} \cdot L(\omega, t)-\frac{t}{2}\left\langle C^{\eta} \hat{\beta}, \hat{\beta}\right\rangle} \approx \\
& \approx\left(\prod_{s=0}^{t}\left(1+\beta \cdot \Delta L^{>\eta}(\omega, s)\right) e^{-\beta \cdot \Delta L^{>\eta}(\omega, s)}\right) e^{-t \beta \cdot \mu_{L}+\beta \cdot L(\omega, t)-\frac{t}{2}\left\langle C^{\eta} \beta, \beta\right\rangle}
\end{aligned}
$$

Observe that if $X=L$, then $\rho(\omega, t)=\mu_{L}$ and $M(\omega, t)=C^{L}$, where as usual $\mu_{L}=\frac{1}{\Delta t} \sum_{a \in A} a p_{a}$ is the drift vector and $C_{i, j}^{L}=\frac{1}{\Delta t} \sum_{a \in A} a_{i} a_{j} p_{a}$ the covariance matrix. The formula above may then be compared to the formulas for diffusions in [5, Theorem 3.5] and for Lévy processes in [4, Section 3].

We have reached the end of the paper, and it may be appropriate to say a few words about the choices I have made. I have chosen to present the theory in the framework of (hyperfinite) Lévy processes, although the basic idea (integrands acting nonlinearly on the increments of the underlying process) is much more general. The main reason is that the existing theory for hyperfinite Lévy processes - and particularly the part relating to splitting infinitesimals - makes it possible to reach interesting results quickly and without too much effort. On the other hand, the interplay between the continuous and the discontinuous is particularly subtle for Lévy processes, and it is reasonable to believe that if we are able to treat the Lévy case, the methods are sufficiently robust to be of general interest.

\section{References}

[1] S. Albeverio, J.E. Fenstad, R. Høegh-Krohn, and T. Lindstrøm: Nonstandard Methods in Stochastic Analysis and Mathematical Physics, Academic Press, New York, 1986.

[2] S. Albeverio and F.S. Herzberg: On an internal random walk representation of measurable Lévy processes and their stochastic integrals, Preprint, 2004 [http://www.maths.ox.ac.uk/ herzberg/]

[3] D. Applebaum: Lévy Processes and Stochastic Calculus, Cambridge University Press, New York, 2004.

[4] T. Chan: Pricing contingent claims on stocks driven by Lévy processes, Ann. Appl. Prob. 9 (1999), 504-528

[5] H. Föllmer and M. Schweizer: Hedging of contingent claims under incomplete information, in Applied Stochastic Analysis, Stochastics Monographs 5, M.H.A. Davis and R.J. Elliott (eds.), Gordon and Breach, New York, 1991, 389-414. 
[6] D.N. Hoover and E.A. Perkins: Nonstandard construction of the stochastic integral and applications to stochastic differential equations I-II, Trans. Amer. Math. Soc. 275 (1983), 1-58.

[7] T. Lindstrøm: Hyperfinite stochastic integration I-III, Math. Scand. 46 (1980), 265-333.

[8] T. Lindstrøm: Internal martingales and stochastic integration, in Nonstandard Analysis:Theory and Applications, L. Arkeryd et al (eds.), Kluwer, Dordrecht, 1997, 209-259.

[9] T. Lindstrøm: Hyperfinite Lévy processes, Stochastics and Stochastics Reports 76 (2004), 517-548.

[10] A.V. Melnikov and A.N. Shiryaev: Criteria for the absence of arbitrage in the financial market, in Frontiers in Pure and Applied Probability II, A.N. Shiryaev et. al (eds.), TVP Science Publishers, Moscow, 1996, 121-134

[11] S-A. Ng: A nonstandard Lévy-Khintchine formula and Lévy processes, Preprint, University of Kwa-Zulu-Natal, 2005, [http://saturn.cs.unp.ac.za/ siuahn/nsalevykhintchin.pdf]

[12] B. Øksendal and A. Sulem: Applied Stochastic Control of Jump Diffusions, Springer, Berlin, 2005.

[13] P. Protter: Stochastic Integration and Differential Equations, 2nd Edition, Springer, Berlin, 2004

[14] M. Schweizer: Variance-optimal hedging in discrete time, Math. Oper. Res. 20 (1995), 1-32

[15] M. Schweizer: On the minimal martingale measure and the FöllmerSchweizer decomposition, Stoch. Anal. Appl. 13 (1995), 573-599 